RU99-11-B

UM-TH-99-11

hep-ph/9912409

\title{
The Transition Temperature to the Superconducting Phase of QCD at High Baryon Density
}

\author{
William E. Brown ${ }^{a}$, James T. Liu ${ }^{b}$ and Hai-cang Ren ${ }^{a, c}$ \\ ${ }^{a}$ Department of Physics, The Rockefeller University, \\ 1230 York Avenue, New York, NY 10021. \\ ${ }^{b}$ Randall Laboratory of Physics, University of Michigan, \\ Ann Arbor, MI 48109. \\ ${ }^{c}$ Department of Natural Science, Baruch College of CUNY, \\ New York, NY 10010.
}

\begin{abstract}
Recent interest in the study of color superconductivity has focused on the regime of high baryon density where perturbative QCD may be employed. Based on the dominant one-gluon-exchange interaction, both the transition temperature and zero temperature gap have been determined to leading order in the coupling, $g$. While the leading non-BCS behavior, $T_{C} \sim \mu g^{-5} e^{-\kappa / g}$, is easily obtained, the pre-exponential factor has proved more difficult to evaluate. Focusing on the transition temperature, we present a perturbative derivation of this factor, exact to leading order in $g$. This approach is first motivated by the study of a toy model and involves working to second order in the perturbative expansion. We compare this result to the zero temperature gap. Additionally, we extend the analysis to the case of higher angular momentum for longitudinal and transverse quark pairing.
\end{abstract}




\section{INTRODUCTION.}

Previous studies of the quark-gluon plasma at high baryon density have indicated the possibility of a pairing instability which may induce a novel superconducting phase of QCD [1] 3]. Based on conventional superconductivity theory, it is known that even the weakest attractive interaction between quarks would be sufficient to form a superconducting pair; for $S U(3)_{c}$ QCD this is provided by the $\overline{\mathbf{3}}$ channel in the diquark interaction. Motivated by studies of QCD under unusual conditions, interest in this phenomenon has recently been revived [4,5]. In fact the phase structure of matter in this regime has important physical consequences for the behaviour of dense astrophysical objects as well as for heavy ion collisions and has raised fundamental questions about the nature of QCD.

In general, for $S U(N)_{c}$ QCD with $N_{f}$ light flavors, the combined symmetry of the theory is initially $S U(N)_{c} \times S U\left(N_{f}\right)_{L} \times S U\left(N_{f}\right)_{R} \times U(1)_{B}$. However, a diquark condensate, $\langle q q\rangle$, would necessarily carry both color and flavor charge, and hence break the above symmetry. The pattern of symmetry breaking is especially interesting for the case of three flavours and colors, in which case the initial $S U(3)_{c} \times S U(3)_{L} \times S U(3)_{R} \times U(1)_{B}$ symmetry is broken to the global diagonal subgroup $S U(3)_{c+L+R}$ [6-10]. Since the condensate remains symmetric under combined color and flavor rotations, it "locks" the color and flavor degrees of freedom. Further interest in the color-flavor locked state has arisen from the observation that many properties such as quantum numbers and roughly similar masses are shared by the bosons produced by this symmetry breaking and hadronic matter, suggesting a connection between these two phases of QCD [7]. These results have led to further investigation of the color-flavor locked phase [11] and the development of an effective field theory for the Nambu-Goldstone bosons produced by the symmetry breaking [12 14.

The nature of the phase transition to the quark gluon plasma and the hadronic phases of QCD has also been studied. Due to competition between confinement and chiral condensation on one side and the formation of a diquark condensate on the other, it is expected that the transition to the hadronic phase is of first order [4,15]18]. Furthermore the phase transition to the quark gluon plasma has also been identified as first order [15, 18]. Other physical implications that have been studied include the effect of differing densities of $s$ compared to $u$ and $d$ quarks, [19, and the effect of a color-superconducting core upon the magnetic field of neutron stars, observed to be stable over very long time scales [20 23].

Since the study of color superconductivity involves working at non-zero chemical potential, it has not been amenable to numerical simulations on a lattice. Instead, a number of analytic methods have been developed to qualitatively probe the color superconducting state [4 6, 8, 15, 16,24, 27]. All of these approaches, to some extent, have difficulties with possible Ansatz dependence, gauge invariance, the Meissner effect as well as the non-linearity of the gap equation, thus making calculations of the condensate properties or the transition temperature somewhat difficult. The consensus of all these models, however, is that, as expected, the strength of the quark-quark interaction in the most attractive channel is of fundamental importance in determining the size of the condensate and hence the properties of the superconducting state [18].

Quantitative results on color superconductivity may be obtained in the limit of ultrahigh baryon density, where perturbative QCD is expected to be valid. In this regime, the dominant attractive interaction arises by single gluon exchange and may be incorporated in 
a Schwinger-Dyson analysis to yield either superconducting gap equations or conditions on the transition temperature. Because of the long range nature of the single gluon exchange interaction, the coupling dependence of the gap $\Delta$ takes on the non-BCS form [28],

$$
\Delta \sim c \frac{\mu}{g^{5}} e^{-\kappa / g}
$$

and this behavior holds for the transition temperature $T_{C}$ as well. In this paper we refine the analysis of the transition temperature and present an exact calculation of $T_{C}$ to leading order in the coupling of the exponential $(\kappa)$ and pre-exponential $(c)$ factors. This is carried out for both longitudinal (LL and RR) and transverse (LR) quark pairings of arbitrary angular momentum, $J$. We find that the $s$-wave $(J=0)$ channel, in which only longitudinal pairings of quarks may occur, is dominant. For $S U(N)_{c}$ QCD the general result takes the form,

$$
\pi k_{B} T_{C}^{J}=\mathcal{C}_{1} \mathcal{C}_{2}^{J} 256 \pi^{4}\left(\frac{2}{N_{f}}\right)^{\frac{5}{2}} \frac{\mu}{g^{5}} e^{-\sqrt{\frac{6 N}{N+1}} \frac{\pi^{2}}{g}},
$$

where $N_{f}$ is the number of flavors, $\mu$ is the chemical potential and $g$ is the running coupling constant evaluated at $\mu$. The largest factor,

$$
\Delta_{0}=256 \pi^{4}\left(\frac{2}{N_{f}}\right)^{\frac{5}{2}} \frac{\mu}{g^{5}} e^{-\sqrt{\frac{6 N}{N+1}} \frac{\pi^{2}}{g}},
$$

was first calculated in [28 30], initially using an elegant renormalisation group analysis. The pre-factors $\mathcal{C}_{1}$ and $\mathcal{C}_{2}^{J}$ represent angular momentum channel independent and dependent corrections respectively, the calculation of which are the focus of this paper. This novel non-BCS dependence of $\Delta_{0}$ on the coupling has been confirmed in 31 33. In addition, the leading order ratio of the transition temperature to the zero temperature gap (in the $s$-wave channel) was investigated in [33] and found to be identical to the BCS case, namely $k_{B} T=\frac{e^{\gamma}}{\pi} \Delta$ where $\gamma=0.5772 \ldots$ is the Euler constant.

In order to exactly determine the pre-factors $\mathcal{C}_{1}$ and $\mathcal{C}_{2}^{J}$, which are of $\mathcal{O}(1)$, it is most straightforward to approach the transition temperature from the normal phase rather than from within the superphase. In the normal phase there is no condensate, no Meissner effect, the problem is linear and there is an established framework to ensure gauge invariance. Following the formulation developed in [34] for the non-relativistic Fermi gas, the DysonSchwinger equation for the scattering of two quarks can be cast into a general eigenvalue problem. This is outlined in [31] and more details are given in this paper. The general eigenvalue problem can be solved to give a set of eigenvalues, $\left\{\lambda_{j}^{2}\right\}$, in terms of the parameters of the theory. The transition to the superphase is then characterised by the locus on the phase diagram where the smallest eigenvalue becomes one. Physically, this condition defines the pole in the solution to the integral equation which represents the formation of a bound state of quarks.

The pre-factor $\mathcal{C}_{1}$ is found to be of $\mathcal{O}(1)$, and has the form:

$$
\begin{aligned}
\mathcal{C}_{1} & =2 e^{\gamma} \exp \left[-\frac{1}{16}\left(\pi^{2}+4\right)(N-1)\right] \\
& \simeq 0.629 \quad \text { for } N=3 .
\end{aligned}
$$


To understand the physical origins of this factor, it can be decomposed into three parts, $\mathcal{C}_{1}=c_{1}^{\prime} c_{1}^{\prime \prime} c_{1}^{\prime \prime \prime}$, each of which are entirely due to single gluon exchange. Higher order diagrams have been shown not to contribute to the leading order of the exponential or the pre-factor in [31]. The first contribution, $c_{1}^{\prime}$, originates in the radiative corrections to the quark propagator due to its self-energy [31],

$$
c_{1}^{\prime}=\exp \left[-\frac{1}{16}\left(\pi^{2}+4\right)(N-1)\right] .
$$

The remaining two contributions, $c_{1}^{\prime \prime}$ and $c_{1}^{\prime \prime \prime}$, are related to the next-to-leading order logarithmic infrared behaviour overlooked in the obtention of (1.3). The factor $c_{1}^{\prime \prime}$ accounts for the sub-leading behaviour lost in the transition from the Matsubara sum at finite temperature to a continuous energy integral,

$$
c_{1}^{\prime \prime}=2 e^{\gamma} .
$$

The final factor, $c_{1}^{\prime \prime \prime}$, represents the contribution of all other approximations made in the obtention of (1.3). Perhaps somewhat surprisingly, we find no such contributions to leading order in the coupling, so that in fact

$$
c_{1}^{\prime \prime \prime}=1 .
$$

The second pre-factor, $\mathcal{C}_{2}^{J}$, classifies the dependence of the critical temperature upon the angular momentum of the pairing. The dependence upon angular momentum is different for longitudinal and transverse condensates. In the dominant $s$-wave channel, for longitudinal pairs we find $\mathcal{C}_{2}^{J=0}=1$ whereas, due to conservation of angular momentum, for transverse pairs we find $\mathcal{C}_{2}^{J=0}=0$. Both longitudinal and transverse pairs may exist for higher angular momentum and the results are summarised below,

$$
\mathcal{C}_{2}^{J}= \begin{cases}\exp \left[3 c_{J}\right] & \text { longitudinal pairs, } J \geq 0, \\ \exp \left[\frac{3}{2}\left(c_{J}+\frac{J}{2 J+1} c_{J+1}+\frac{J+1}{2 J+1} c_{J-1}\right)\right] & \text { transverse pairs, } J \geq 1,\end{cases}
$$

where,

$$
c_{J}= \begin{cases}0 & \text { for } J=0, \\ -2 \sum_{n=1}^{J} \frac{1}{n} & \text { for } J \neq 0 .\end{cases}
$$

This indicates the suppression of the higher angular-momentum channels, so that $s$-wave pairing is dominant and $p$-wave pairing is already down by about three orders of magnitude.

Before proceeding to the complete derivation of (1.2), we shall first illustrate the perturbative formalism in section II and apply it to an exactly soluble toy model. Then in section III, the Dyson-Schwinger equation for the interaction between a longitudinal pair of quarks of zero angular momentum is studied in the Coulomb gauge. The resulting Fredholm equation is cast into a general eigenvalue problem. A perturbative solution for this eigenvalue problem is found in section IV. Systematic calculation to second order in this perturbation series captures all of the double and linear logarithmic infrared behaviour of the eigenvalue that determines (1.2) to leading order in the coupling. The generalization to non-zero angular momentum and transverse pairings is made in section $\mathrm{V}$ and in section VI we discuss numerical verification and the gauge invariance of our results. Concluding remarks are made in section VII. 


\section{A TOY MODEL: THE TWO-DIMENSIONAL CYLINDRICAL WELL.}

In this section we shall develop the perturbative formalism that will be used in the following sections. As shown in [31, the Schwinger-Dyson equation determining the transition temperature has the form of a Fredholm integral equation and may be re-cast as an eigenvalue problem. We thus seek a systematic perturbative method of developing the eigenvalues of the Fredholm equation. To illustrate this method, we begin by considering the Schrodinger problem for a two-dimensional cylindrical well of depth $\kappa^{2} / a^{2}$ and radius $a$ :

$$
\left(\frac{\partial^{2}}{\partial x_{i}^{2}}+\frac{\kappa^{2}}{a^{2}} \theta(a-|\vec{x}|)\right) \psi(\vec{x})=\Delta^{2} \psi(\vec{x})
$$

(where we have normalised the mass so that $2 m=1$ ). As a toy model, it is easily solved in terms of Bessel functions and matching conditions at the well boundary. On the other hand, it may also be converted into an integral equation of Fredholm type. The perturbative solution to the resulting Fredholm equation will serve the basis for generalization to the real problem of determining the QCD transition temperature.

Note that although two parameters appear in (2.1), namely $a$ and $\kappa$, only the dimensionless coupling $\kappa$ is physical, as $a$ may be scaled away by $\vec{x} \rightarrow a \vec{x}$. In analogy with the QCD problem, $\kappa$ represents the QCD coupling $g$, and $a$ represents the inverse of the chemical potential $\mu$. The eigenvalue $\Delta^{2}$ plays the rôle of either the critical temperature or the mass gap of the color superconductor and in the weak coupling limit has the form $\Delta \propto(1 / a) \exp \left(-1 / \kappa^{2}\right)$. While this behavior is BCS-like (as may be deduced from the shortrange nature of the cylindrical well potential) some mathematical features of this toy model nevertheless extend to the long-range QCD case.

In the following, we show that this leading order in $\kappa$ behaviour of $\Delta$ can be recovered from a perturbative expansion of the resulting Fredholm kernel, where the zeroth order operator is defined to capture the leading logarithmic behaviour. In this case we find that the exponential and its pre-factor can be determined to leading order in $\kappa$ by second order perturbation theory. Furthermore, the exponential is determined by the leading logarithmic behaviour and the pre-factor is determined by the sub-leading behaviour uncovered by perturbation theory.

For comparison with the perturbative method, we now briefly give the exact solution of the model. From the rotational symmetry of the problem, only the $s$-wave equation is important. In this case the radial Schrodinger equation becomes

$$
\frac{1}{r} \frac{d}{d r}\left(r \frac{d \psi(r)}{d r}\right)+\frac{\kappa^{2}}{a^{2}} \theta(a-r) \psi(r)=\Delta^{2} \psi(r) .
$$

The solutions of (2.2) are Bessel functions, both inside and outside the well. The eigenvalue problem then results from the matching conditions at $r=a$. For a bound state, $\Delta^{2}>0$, the matching conditions read,

$$
\sqrt{\kappa^{2}-\Delta^{2} a^{2}} \frac{J_{1}\left(\sqrt{\kappa^{2}-\Delta^{2} a^{2}}\right)}{J_{0}\left(\sqrt{\kappa^{2}-\Delta^{2} a^{2}}\right)}=\Delta a \frac{K_{1}(\Delta a)}{K_{0}(\Delta a)} .
$$


To draw out the similarities with superconductivity, we find the leading order solution in the weak coupling limit, $\Delta a \ll 1$ and $\kappa \ll 1$, to be

$$
\Delta \simeq \Delta_{0} \exp \left(-\frac{2}{\kappa^{2}}\right)
$$

where $\Delta_{0}=(2 / a) \exp (-\gamma+1 / 4)$.

To make contact with the Fredholm problem, we now re-write the toy model in terms of an integral equation in momentum space. Using the Fourier transform of the potential,

$$
V(\vec{q})=-\frac{\kappa^{2}}{a^{2}} \int d^{2} \vec{x} e^{-i \vec{q} \cdot \vec{x}} \theta(a-|x|)=-2 \pi \kappa^{2} \frac{J_{1}(q a)}{q a},
$$

and wavefunction,

$$
\psi(\vec{x})=\int \frac{d^{2} \vec{p}}{(2 \pi)^{2}} e^{i \vec{p} \cdot \vec{x}} \chi(\vec{p})
$$

we get a homogeneous Fredholm equation similar to that obtained from the Schwinger-Dyson approach to QCD at high density,

$$
\chi(\vec{p})=-\frac{1}{p^{2}+\Delta^{2}} \int \frac{d^{2} \vec{q}}{(2 \pi)^{2}} V(\vec{p}-\vec{q}) \chi(\vec{q}) .
$$

Partial wave analysis simplifies the solution of this integral equation. Expanding the potential in the standard way,

$$
\begin{aligned}
V(\vec{p}-\vec{q}) & =\sum_{m} V_{m}(p, q) e^{i m \phi}, \\
V_{m}(p, q) & =\frac{1}{2 \pi} \int_{0}^{2 \pi} d \phi e^{-i m \phi} V(\vec{p}-\vec{q})=-\frac{\kappa^{2}}{a^{2}} 2 \pi \int_{0}^{a} d r r J_{m}(p r) J_{m}(q r),
\end{aligned}
$$

we note, for a cylindrically symmetric wavefunction $\chi(p)$, that only $V_{0}$ survives the integration over the azimuthal angle in (2.7) and we have

$$
\chi(p)=-\frac{1}{2 \pi\left(p^{2}+\Delta^{2}\right)} \int_{0}^{\infty} d q q V_{0}(p, q) \chi(q) .
$$

We now consider the general eigenvalue problem,

$$
\chi(\vec{p})=-\frac{\lambda^{2}}{p^{2}+\Delta^{2}} \int \frac{d^{2} \vec{q}}{(2 \pi)^{2}} V(\vec{p}-\vec{q}) \chi(\vec{q})
$$

for which the condition $\lambda^{2}=1$ identifies the specific problem of (2.7). We shall find solutions, in the weak coupling limit, for the eigenvalues $\lambda^{2}$ in terms of the parameters of the theory; $\kappa, a$ and $\Delta$.

Perturbatively it is convenient to work with a real and symmetric kernel. Noting that $V_{0}(p, q)$ is already symmetric in $p$ and $q$ and that the kernel is real, symmetrization is achieved with the definition, 


$$
f(p)=\sqrt{p\left(p^{2}+\Delta^{2}\right)} \chi(p),
$$

so that the problem is reduced to the solution of the integral equation,

$$
f(p)=\lambda^{2} \int_{0}^{\infty} d q \mathcal{K}_{S}(p, q) f(q)
$$

where

$$
\mathcal{K}_{S}(p, q)=-\frac{1}{2 \pi} \sqrt{\frac{p q}{\left(p^{2}+\Delta^{2}\right)\left(q^{2}+\Delta^{2}\right)}} V_{0}(p, q)
$$

is the symmetrized kernel.

We observe that the most divergent region of $\mathcal{K}_{S}$ is at small $p$ and $q$. Since $V_{0}$ is well behaved at small $p$ and $q$ the dangerous terms arise in the denominator of (2.13) and are damped by $\Delta$. Thus the important contributions to the integral equation arise in the region $\{p, q\} \approx \Delta$. To highlight this region, we split the integral equation into two parts by introducing a scale $\delta$ such that $\Delta \ll \delta \ll 1 / a$. We first look at the region $p<\delta, q<\delta$ that contains the leading order behaviour. In this region, $V_{0}(p, q) \simeq-\kappa^{2} \pi$, so the kernel may be split into a zeroth order term and correction:

$$
\mathcal{K}_{S}(p, q)=\mathcal{K}_{S}^{\circ}(p, q)+\mathcal{K}_{S}^{\prime}(p, q)
$$

where

$$
\mathcal{K}_{S}^{\circ}(p, q)=\frac{\kappa^{2}}{2} \sqrt{\frac{p q}{\left(p^{2}+\Delta^{2}\right)\left(q^{2}+\Delta^{2}\right)}} \theta(\delta-p) \theta(\delta-q)
$$

and

$$
\mathcal{K}_{S}^{\prime}(p, q)=\frac{\kappa^{2}}{a^{2}} \sqrt{\frac{p q}{\left(p^{2}+\Delta^{2}\right)\left(q^{2}+\Delta^{2}\right)}} \int_{0}^{a} d r r\left[J_{0}(p r) J_{0}(q r)-\theta(\delta-p) \theta(\delta-q)\right] .
$$

To the zeroth order, the integral equation (2.12) reduces to

$$
f^{\circ}(p)=\lambda^{\circ 2} \int_{0}^{\delta} d q \mathcal{K}_{S}^{\circ}(p, q) f^{\circ}(q)
$$

We find that the only nonzero eigenvalue is given by

$$
\frac{1}{\lambda^{\circ 2}} \simeq \frac{\kappa^{2}}{2} \log \frac{\delta}{\Delta}
$$

with the corresponding (normalized) eigenfunction

$$
f^{\circ}(p)=\frac{1}{\sqrt{\log (\delta / \Delta)}} \sqrt{\frac{p}{p^{2}+\Delta^{2}}} \theta(\delta-p) .
$$

To obtain a perturbative solution to the full eigenvalue problem, we introduce a complete set of orthonormal functions, $\langle p \mid m\rangle$ where $m=0,1,2, \ldots$ labels the eigenstate and $|0\rangle$ 
corresponds to the state $(2.19)$, i.e. $\langle p \mid 0\rangle=f^{\circ}(p)$. Using the completeness relation $1=$ $\sum_{m}|m\rangle\langle m|$ and the fact that the states $|m\rangle$ for $m>0$ have zero eigenvalue under the zeroth order kernel, the eigenvalue for the full kernel can then be calculated to second order,

$$
\frac{1}{\lambda^{2}}=\frac{1}{\lambda^{\circ}}+\left\langle 0\left|\mathcal{K}_{S}^{\prime}\right| 0\right\rangle+\lambda^{\circ 2}\left\langle 0\left|\mathcal{K}_{S}^{\prime 2}\right| 0\right\rangle-\lambda^{\circ 2}\left\langle 0\left|\mathcal{K}_{S}^{\prime}\right| 0\right\rangle^{2}
$$

where the inner product is defined by, for example,

$$
\left\langle 0\left|\mathcal{K}_{S}^{\prime}\right| 0\right\rangle=\int_{0}^{\infty} d q \int_{0}^{\infty} d p f^{\circ}(p) \mathcal{K}_{S}^{\prime}(p, q) f^{\circ}(q) .
$$

To determine the sub-leading behaviour of (2.20) relative to (2.18) we are looking for contributions to the eigenvalue of $\mathcal{O}(1)$. Since we are in the weak coupling limit, expansion of the Bessel functions in (2.16) quickly leads to the result,

$$
\left\langle 0\left|\mathcal{K}_{S}^{\prime}\right| 0\right\rangle \simeq \mathcal{O}\left(\frac{1}{\log (\delta / \Delta)}\right)
$$

so that the first order correction is found to be insignificant. This also implies that the last term of (2.20) can be discarded. We are then left with one term to calculate at second order. Although the zeroth order wavefunctions restrict the initial and final momenta to lie in the range $[0, \delta]$, the intermediary momentum in $\left\langle 0\left|\mathcal{K}_{S}^{\prime 2}\right| 0\right\rangle$ (resulting from the convolution of the kernel) is free to take all possible values $[0, \infty)$. Although no small argument expansion of Bessel functions with the intermediary momentum as an argument is allowed, the resulting integrals can be performed exactly and give,

$$
\lambda^{\circ 2}\left\langle 0\left|\mathcal{K}_{S}^{\prime 2}\right| 0\right\rangle=\frac{\kappa^{2}}{2}\left(\log \left(\frac{2}{\delta a}\right)-\gamma+\frac{1}{4}\right) .
$$

Substituting (2.18), (2.22) and (2.23) into (2.20) we find that the dependence upon the arbitrary scale $\delta$ that appears at zeroth order is cancelled by the second order correction, as is

required. The leading and sub-leading behaviour of the eigenvalue has thus been calculated to be

$$
\frac{1}{\lambda^{2}} \simeq \frac{\kappa^{2}}{2}\left(\log \left(\frac{2}{\Delta a}\right)-\gamma+\frac{1}{4}\right)
$$

From the condition $\lambda^{2}=1$, the result (2.4) is recovered.

\section{THE FREDHOLM EQUATION FOR THE TRANSITION TEMPERATURE.}

We now return to the problem at hand and consider an $S U(N)_{c}$ color gauge field coupling to $N_{f}$ flavors of massless quarks with the Lagrangian density,

$$
\mathcal{L}=-\frac{1}{4} F_{\mu \nu}^{a} F_{\mu \nu}^{a}-\bar{\psi}_{f} \gamma_{\mu}\left(\partial_{\mu}-i g A_{\mu}\right) \psi_{f}
$$




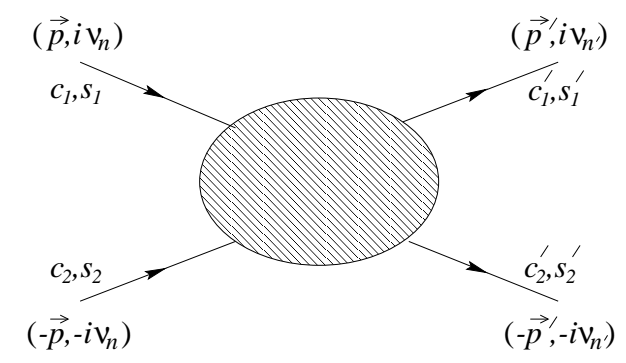

FIG. 1. Proper vertex function, $\Gamma_{s_{1}^{\prime}, s_{2}^{\prime} ; s_{1}, s_{2}}^{c_{1}^{\prime}, c^{\prime} ; c_{1}, c_{2}}\left(n^{\prime}, \vec{p}^{\prime} \mid n, \vec{p}\right)$.

where $F_{\mu \nu}^{a}=\partial_{\mu} A_{\nu}^{a}-\partial_{\nu} A_{\mu}^{a}+g f^{a b c} A_{\mu}^{b} A_{\nu}^{c}$ and $A_{\mu}=A_{\mu}^{a} t^{a}$ with $t^{a}$ the $S U(N)_{c}$ generator in its fundamental representation. Since the Lagrangian (3.1) is diagonal with respect to both flavor and chirality, the corresponding indices will be dropped below.

As in [31], we shall study the proper vertex function, $\Gamma$, corresponding to the scattering of two quarks with zero total energy and momentum. Our conventions are the same as in [31] but are repeated here for clarity of presentation. The Matsubara energies $i \nu_{n}$ of individual ingoing and outgoing quarks are labelled by $n$ and $n^{\prime}$, respectively. Similarly, $\vec{p}$ and $\vec{p}^{\prime}$ label the ingoing and outgoing momenta. Each of the superscripts $c_{i}, i=1,2$, denote the color associated with each leg and the subscripts $s$, which label the states above or below the Dirac sea, are either + or - . Primed variables are outgoing and unprimed incoming. The ingoing quark pair are labelled by $s=\left(s_{1}, s_{2}\right)$ and $c=\left(c_{1}, c_{2}\right)$ and the outgoing pair by $s^{\prime}=\left(s_{1}^{\prime}, s_{2}^{\prime}\right)$ and $c^{\prime}=\left(c_{1}^{\prime}, c_{2}^{\prime}\right)$. This labelling is illustrated in Fig. $\mathbb{1}$.

The proper four-fermion vertex function satisfies a Schwinger-Dyson equation (shown in Fig. 2) which, with all indices suppressed, may be written,

$$
\Gamma\left(n^{\prime}, \vec{p}^{\prime} \mid n, \vec{p}\right)=\tilde{\Gamma}\left(n^{\prime}, \vec{p}^{\prime} \mid n, \vec{p}\right)+\frac{1}{\beta} \sum_{m} \int \frac{d^{3} \vec{q}}{(2 \pi)^{3}} K\left(n^{\prime}, \vec{p}^{\prime} \mid m, \vec{q}\right) \Gamma(m, \vec{q} \mid n, \vec{p}),
$$

where $\tilde{\Gamma}$ represents the two quark irreducible vertex. The kernel has the explicit form,

$$
K_{s_{1}^{\prime}, s_{2}^{\prime} ; s_{1}, s_{2}}^{c_{1}^{\prime}, c_{2}^{\prime}, c_{1}, c_{2}}\left(n^{\prime}, \vec{p}^{\prime} \mid m, \vec{q}\right)=\tilde{\Gamma}_{s_{1}^{\prime}, s_{2}^{\prime} ; s_{1}, s_{2}}^{c_{\prime}^{\prime}, c^{\prime} ; c_{1}, c_{2}}\left(n^{\prime}, \vec{p}^{\prime} \mid m, \vec{q}\right) S_{s_{1}}(m \mid \vec{q}) S_{s_{2}}(-m \mid-\vec{q}),
$$

where $S_{s}(n \mid \vec{p})$ denotes the full quark propagator with momentum $\vec{p}$ and Matsubara energy $i \nu_{n}=\frac{2 \pi i}{\beta}\left(n+\frac{1}{2}\right)$. In order to facilitate the partial wave analysis we found it convenient to associate the Dirac spinors $u(\vec{p})$ and $v(\vec{p})$, which satisfy the Dirac equations $\left(\gamma_{4} p-i \vec{\gamma}\right.$. $\vec{p}) u(\vec{p})=0$ and $\left(\gamma_{4} p-i \vec{\gamma} \cdot \vec{p}\right) v(\vec{p})=0$, to the quark-gluon vertex instead of to the quark propagator. Thus the vertices written in (3.2) are of the form,

$$
\Gamma_{s^{\prime} ; s}=\bar{U}_{\gamma}\left(s_{1}^{\prime}, \vec{p}^{\prime}\right) \bar{U}_{\delta}\left(s_{2}^{\prime},-\vec{p}^{\prime}\right) \Gamma_{\gamma \delta, \alpha \beta} U_{\alpha}\left(s_{1}, \vec{p}\right) U_{\beta}\left(s_{2},-\vec{p}\right),
$$

with the vertex function $\Gamma_{\gamma \delta, \alpha \beta}$ given by conventional Feynman rules and $U(+, \vec{p})=u(\vec{p})$ or $U(-, \vec{p})=v(-\vec{p})$, respectively. However, since the quarks are massless, $\gamma_{5} u=-u$ and $\gamma_{5} v=-v$, to simplify notation we may identify $U(s, \vec{p})=u(s \vec{p})$.

We complete this section of conventions and definitions with the fermion and gluon propagators. The zeroth order quark propagator reads, 


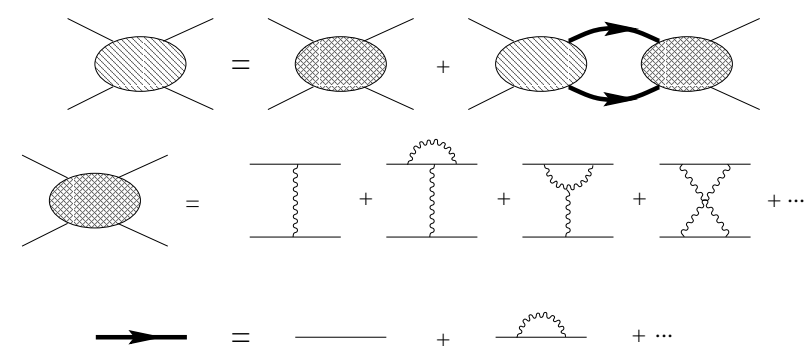

FIG. 2. The Schwinger-Dyson equation. $\Gamma$ is represented by single hashed vertices and $\tilde{\Gamma}$ is represented by double hashed vertices. The full quark propagator is represented by a solid line and the bare quark propagator by a thin line. Gluon propagators, including hard thermal loops, are represented by curly lines. The first two orders in the expansion of $\tilde{\Gamma}$ and the full quark propagator are given.

$$
S_{s}(n \mid \vec{p})=\frac{i}{i \nu_{n}-s p+\mu} .
$$

The radiative corrections to this propagator contribute to the pre-factor $\mathcal{C}_{1}$ in (1.2). Such radiative corrections are depicted in Fig. 2. This contribution has been calculated in a previous publication, [31, and the reader is directed there for details.

We discuss the gauge invariance of this approach to the transition temperature in section VI, but until then we shall work in the Coulomb gauge where the gluon propagator takes the form,

$$
\begin{aligned}
D_{44}(\vec{k}, \omega) & =D^{E}(\vec{k}, \omega), \\
D_{i j}(\vec{k}, \omega) & =D^{M}(\vec{k}, \omega)\left(\delta_{i j}-\frac{k_{i} k_{j}}{\vec{k}^{2}}\right), \\
D_{4 j}(\vec{k}, \omega) & =D_{j 4}(\vec{k}, \omega)=0 .
\end{aligned}
$$

In the presence of a Fermi sea, hard dense loops should be included in the gluon propagator in the leading order. While it is possible that a magnetic mass of order $T$ may exist, hard dense loops lead to Landau damping which prevails at $\mu \gg k_{B} T$ [35]. In general, the screened electric and magnetic propagators take the form,

$$
\begin{aligned}
D^{E}(\vec{k}, \omega) & =\frac{-i}{\vec{k}^{2}+\sigma^{E}(\vec{k}, \omega)}, \\
D^{M}(\vec{k}, \omega) & =\frac{-i}{\vec{k}^{2}+\omega^{2}+\sigma^{M}(\vec{k}, \omega)},
\end{aligned}
$$

In the region $k \ll \mu$ and $\omega \ll \mu$, both $\sigma^{E}(\vec{k}, \omega)$ and $\sigma^{M}(\vec{k}, \omega)$ depend only upon the ratio $x=\omega /|\vec{k}|, x \in(-\infty, \infty)$. Scaling out the Debye mass,

$$
m_{D}^{2}=\frac{N_{f} g^{2}}{\pi^{2}} \int_{0}^{\infty} d q q \frac{1}{e^{\beta(q-\mu)}+1} \simeq \frac{N_{f} g^{2} \mu^{2}}{2 \pi^{2}},
$$

by writing $\sigma^{E}(\vec{k}, \omega)=m_{D}^{2} f_{E}(x)$ and $\sigma^{M}(\vec{k}, \omega)=m_{D}^{2} f_{M}(x)$, we find the dimensionless screening functions, 


$$
\begin{aligned}
f_{E}(x) & =\left[1-x \tan ^{-1}\left(\frac{1}{x}\right)\right], \\
f_{M}(x) & =\frac{x}{2}\left[\left(1+x^{2}\right) \tan ^{-1}\left(\frac{1}{x}\right)-x\right] .
\end{aligned}
$$

Analysis of the functions $f_{E}(x)$ and $f_{M}(x)$ shows that the dangerous region for infrared divergences to originate in the gluon propagator is at small $x$, corresponding to energy and momentum transfer $\omega \ll k \ll \mu$. In this region the gluon self-energy functions are well approximated by

$$
\begin{aligned}
f_{E}(x) & \simeq 1, \\
f_{M}(x) & \simeq \frac{\pi}{4}|x|,
\end{aligned}
$$

indicating that, while the Coulomb interaction is strongly screened by the Debye length, the magnetic interaction is poorly screened. It is for this reason that the attractive interaction responsible for the pairing instability is dominated by single collinear magnetic gluon exchange. Furthermore, $f_{E}(x)$ is a decreasing function of $x$ and $f_{M}(x)$ is an increasing function of $x$, which obey the following inequalities for all $x$ :

$$
\begin{aligned}
f_{E}(x) & \leq 1 \\
f_{M}(x) & \leq \frac{\pi}{4}|x| .
\end{aligned}
$$

For $x \gg 1$ we have,

$$
\begin{aligned}
f_{E}(x) & \simeq \frac{1}{3 x^{2}}, \\
f_{M}(x) & \simeq \frac{1}{3} .
\end{aligned}
$$

Since $\Gamma$ corresponds to di-quark scattering it can be decomposed into irreducible representations of $S U(N)_{c}$ by either symmetrization or antisymmetrization among the initial and final color indices. We decompose $\Gamma$ into its symmetric and anti-symmetric components,

$$
\begin{aligned}
\Gamma_{s^{\prime}, s}^{c^{\prime}, c}\left(n^{\prime}, \vec{p}^{\prime} \mid n, \vec{p}\right) & \left.=\sqrt{2} \delta^{c_{1}\left(c_{1}^{\prime}\right.} \delta^{c_{2}^{\prime}}\right) c_{2} \Gamma_{s^{\prime}, s}^{S}\left(n^{\prime}, \vec{p}^{\prime}, \mid n, \vec{p}\right) \\
& +\sqrt{2} \delta^{c_{1}\left[c_{1}^{\prime}\right.} \delta^{\left.c_{2}^{\prime}\right] c_{2}} \Gamma_{s^{\prime}, s}^{A}\left(n^{\prime}, \vec{p}^{\prime}, \mid n, \vec{p}\right),
\end{aligned}
$$

where $[\cdots]$ and $(\cdots)$ denote antisymmetrization and symmetrization with weight one respectively (and likewise for $\tilde{\Gamma}$ ). Since the Fermi surface has a pairing instability in the presence of even an arbitrarily weak attractive interaction we need only focus on the attractive antisymmetric channel.

Proceeding with the partial wave analysis, we expand $\Gamma_{s^{\prime}, s}^{A}\left(n^{\prime}, \vec{p}^{\prime} \mid n, \vec{p}\right)$ in terms of Legendre polynomials,

$$
\Gamma_{s^{\prime}, s}^{A}\left(n^{\prime}, \vec{p}^{\prime} \mid n, \vec{p}\right)=\sum_{J} \gamma_{s^{\prime}, s}^{J}\left(n^{\prime}, p^{\prime} \mid n, p\right) P_{J}(\cos \theta)
$$

Using a similar expression for $\tilde{\Gamma}_{s^{\prime}, s}^{A}\left(n^{\prime}, \vec{p}^{\prime} \mid n, \vec{p}\right)$, we find from (3.2) the Fredholm equation satisfied by $\gamma_{s^{\prime}, s}^{J}\left(n^{\prime}, p^{\prime} \mid n, p\right)$ : 


$$
\gamma_{s^{\prime}, s}^{J}\left(n^{\prime}, p^{\prime} \mid n, p\right)=\tilde{\gamma}_{s^{\prime}, s}^{J}\left(n^{\prime}, p^{\prime} \mid n, p\right)+\frac{1}{\beta} \sum_{m, s^{\prime \prime}} \int_{0}^{\infty} d q K_{s^{\prime}, s^{\prime \prime}}^{J}\left(n^{\prime}, p^{\prime} \mid m, q\right) \gamma_{s^{\prime \prime}, s}^{J}(m, q \mid n, p)
$$

where the kernel $K_{s^{\prime}, s}^{J}$ has the form,

$$
K_{s^{\prime}, s}^{J}\left(n^{\prime}, p^{\prime} \mid n, p\right)=\frac{p^{2} \tilde{\gamma}_{s^{\prime}, s}^{J}\left(n^{\prime}, p^{\prime} \mid n, p\right)}{2 \pi^{2}(2 J+1)} S_{s_{1}}(n \mid p) S_{s_{2}}(-n \mid p)
$$

This integral equation can be cast into a general eigenvalue problem in the way outlined for the toy model in section II. To do so, we first note that the formal solution of (3.22) involves the Fredholm determinant

$$
\mathcal{D}=\operatorname{det}(1-K)=\prod_{j}\left(1-\lambda_{j}^{-2}\right)
$$

where the eigenvalues, $\left\{\lambda_{j}^{2}\right\}$, are defined by the solutions of,

$$
f_{s}(n, p)=\frac{\lambda^{2}}{\beta} \sum_{n^{\prime}, s^{\prime}} \int_{0}^{\infty} d p^{\prime} K_{s^{\prime}, s}^{J}\left(n^{\prime}, p^{\prime} \mid n, p\right) f_{s^{\prime}}\left(n^{\prime}, p^{\prime}\right)
$$

Since $\mathcal{D}$ appears in the denominator in the inversion of the Fredholm equation, it in fact governs the pole in the solution that represents the formation of a bound state of quarks at the transition point to the superphase. For weak coupling, we may identify the pairing temperature and the phase coherence temperature.

At sufficiently high temperature all of the set of eigenvalues $\left\{\lambda_{j}^{2}\right\}$ are greater than one, so that $\mathcal{D} \neq 0$ and there is no instability - the theory is in the normal phase. As the temperature is reduced, we find the transition temperature to the superphase is that at which the smallest of $\left\{\lambda_{j}^{2}\right\}$ reaches one. If we label the solutions so that the smallest eigenvalue is $\lambda_{0}^{2}$, then the transition temperature is obtained from the inversion of the condition, $\lambda_{0}^{2}\left(T_{C}, g, \mu\right)=1$.

In order to proceed with a second order perturbative calculation, it is easiest to work with a symmetric kernel. In the general eigenvalue problem defined by (3.25) we have a kernel $K_{s^{\prime}, s} \equiv K_{s_{1}^{\prime} s_{2}^{\prime}, s_{1} s_{2}}$, where the leading order behaviour only occurs for particle states (that can see the Fermi sea), namely for $s=(+,+)$ and $s^{\prime}=(+,+)$. In general, however, the solution $f_{++}$is necessarily coupled to solutions $f_{+-}, f_{-+}$and $f_{--}$by the kernels $K_{+-,++}, K_{-+,++}$ and $K_{--,++}$, respectively. On the other hand, since '-' represents a quark below the Dirac sea, its corresponding propagator has no pole for momentum at the Fermi sea, $p=\mu$, as may be seen from (3.5). In fact, the absence of this pole is sufficient to remove two orders of the logarithm and hence the cross-channels make no contribution to either the leading or the subleading behaviour. In the following we shall focus on the kernel $K=K_{++,++}$and drop all particle/antiparticle notation.

The kernel $K$ is symmetric under simultaneous switch of the sign of the Matsubara labels $n^{\prime}$ and $n$, i.e., $K\left(n^{\prime}, p^{\prime} \mid n, p\right)=K\left(-n^{\prime}, p^{\prime} \mid-n, p\right)$. As a result, the eigenfunctions $f(n, p)$ must then be either even or odd with respect to $n$. Since only the even functions pick up the singularities on the Fermi surface we shall concentrate on them and replace the sum $\sum_{n=-\infty}^{\infty}$ by $\sum_{n=0}^{\infty}$.

Symmetrization of the problem proceeds by identifying 


$$
f(n, p)=\frac{\sqrt{\nu_{n}^{2}+(p-\mu)^{2}}}{p} h\left(\nu_{n}, p\right) .
$$

Then from (3.25) and (3.23) and using $h\left(\nu_{n}, p\right)=h\left(-\nu_{n}, p\right)$ we find,

$$
h\left(\nu_{n}, p\right)=\frac{\lambda^{2}}{\beta} \sum_{n^{\prime}=0}^{\infty} \int_{0}^{\infty} d p^{\prime} K_{S}^{J}\left(n^{\prime}, p^{\prime} \mid n, p\right) h\left(\nu_{n^{\prime}}, p^{\prime}\right),
$$

where the symmetrized kernel, $K_{S}^{J}$, is written,

$$
K_{S}^{J}\left(n^{\prime}, p^{\prime} \mid n, p\right)=-\frac{p p^{\prime}}{2 \pi^{2}(2 J+1)}\left\{\frac{\tilde{\gamma}^{J}\left(n^{\prime}, p^{\prime} \mid n, p\right)+\tilde{\gamma}^{J}\left(-n^{\prime}, p^{\prime} \mid n, p\right)}{\sqrt{\left(\nu_{n}^{2}+(p-\mu)^{2}\right)\left(\nu_{n^{\prime}}^{2}+\left(p^{\prime}-\mu\right)^{2}\right)}}\right\} .
$$

This will be the starting point for the perturbative calculation of the next section.

\section{PERTURBATIVE CALCULATION OF THE TRANSITION TEMPERATURE.}

In this section we shall concentrate upon the calculation of the transition temperature for a longitudinal pair in the $s$-wave channel, $J=0$. The discussion of the transition temperatures corresponding to higher angular momenta longitudinal and transverse pairings will be deferred until section $\mathrm{V}$. To zeroth order in the perturbative expansion, we start with the leading order solution obtained previously in 28 33. In the following subsections we shall define and evaluate a perturbation series to uncover the subleading behaviour.

For one gluon exchange, incorporating hard dense loops in the bare quark propagators, we find (in the $K_{++,++}$channel),

$$
\begin{aligned}
\tilde{\gamma}^{J=0}\left(n^{\prime}, p^{\prime} \mid n, p\right)=-\frac{g^{2}}{8} & \left(1+\frac{1}{N}\right) \int_{-1}^{1} d(\cos \theta) \\
& \times\left[\frac{3-\cos \theta-(1+\cos \theta) \frac{\left(p-p^{\prime}\right)^{2}}{\left|\vec{p}-\vec{p}^{\prime}\right|^{2}}}{\left(\nu_{n}-\nu_{n^{\prime}}\right)^{2}+\left|\vec{p}-\vec{p}^{\prime}\right|^{2}+m_{D}^{2} f_{M}(x)}+\frac{1+\cos \theta}{\left|\vec{p}-\vec{p}^{\prime}\right|^{2}+m_{D}^{2} f_{E}(x)}\right]
\end{aligned}
$$

where $x=\left(\nu_{n}-\nu_{n^{\prime}}\right) /\left|\vec{p}-\vec{p}^{\prime}\right|$. The first term in the integrand arises from magnetic gluon exchange and the second from electric gluon exchange. Further discussion of this vertex is contained in appendix A. To obtain the leading double logarithmic behaviour we see that the infrared-sensitive region is in the forward direction, $\theta \approx 0$, so that $\cos \theta$ may be approximated by one in the numerator of the integrand. Furthermore, we discard the energy transfer term, $\left(\nu_{n}-\nu_{n^{\prime}}\right)^{2}$, in the denominator of the magnetic propagator and approximate the self-energy functions, $f_{E}(x)$ and $f_{M}(x)$, by their asymptotic forms (3.14) and (3.15) for small $x$. These approximations, prevalent in the literature, over-estimate both the electric and magnetic screening and thus under-estimate the attraction between quarks. How this correlates with the transition temperature is hard a priori to quantify. Although a weaker attraction should lead to a lower transition temperature and smaller condensates we shall see that, in fact, none of the corrections to these leading order approximations discard any next-to-leading behaviour of the eigenvalue. 
With these approximations, the integration over $\cos \theta$ becomes trivial,

$$
\tilde{\gamma}^{J=0}\left(n, p \mid n^{\prime}, p^{\prime}\right) \simeq-\frac{g^{2}}{12 p p^{\prime}}\left(1+\frac{1}{N}\right)\left[\log \frac{8 \mu^{3}}{\left|p-p^{\prime}\right|^{3}+\frac{\pi}{4} m_{D}^{2}\left|\nu_{n}-\nu_{n^{\prime}}\right|}+\frac{3}{2} \log \frac{4 \mu^{2}}{m_{D}^{2}}\right] .
$$

To leading order, we may also neglect the momentum exchange under the first logarithm. Although this produces a logarithmic singularity for $n=n^{\prime}$ which is potentially dangerous, we shall see that in fact it is well behaved. Combining the electric and magnetic parts we find,

$$
\tilde{\gamma}^{J=0}\left(n^{\prime}, p^{\prime} \mid n, p\right) \simeq-\frac{g^{2}}{12 p p^{\prime}}\left(1+\frac{1}{N}\right) \log \frac{1}{\left|\hat{\nu}_{n}-\hat{\nu}_{n^{\prime}}\right|}
$$

where

$$
\hat{\nu}=\frac{g^{5}}{256 \pi^{4}}\left(\frac{N_{f}}{2}\right)^{\frac{5}{2}} \frac{\nu}{\mu}
$$

Using the Ansatz,

$$
h^{\circ}\left(\nu_{n}, p\right)=\frac{\chi\left(\nu_{n}\right)}{\sqrt{\nu_{n}^{2}+(p-\mu)^{2}}},
$$

and substituting into (3.27), we find that the leading order eigenvalue problem becomes,

$$
\chi_{j}\left(\nu_{n}\right)=\lambda_{j}^{\circ} \frac{g^{2}}{24 \pi^{2}}\left(1+\frac{1}{N}\right) \frac{1}{\beta} \sum_{n^{\prime}=0}^{\infty} \int_{0}^{\infty} d p^{\prime} K^{\circ}\left(n^{\prime}, p^{\prime} \mid n, p\right) \sqrt{\frac{\nu_{n}^{2}+(p-\mu)^{2}}{\nu_{n^{\prime}}^{2}+\left(p^{\prime}-\mu\right)^{2}}} \chi_{j}\left(\nu_{n}^{\prime}\right)
$$

where the kernel focuses upon the most divergent region of the Matsubara energies, and we impose a cut, $\hat{\nu}<\hat{\delta}$, analogous to that used for the toy model in section II:

$$
K^{\circ}\left(n^{\prime}, p^{\prime} \mid n, p\right)=\left\{\frac{\log \frac{1}{\left|\hat{\nu}_{n}-\hat{\nu}_{n^{\prime}}\right|}+\log \frac{1}{\left|\hat{\nu}_{n}+\hat{\nu}_{n^{\prime}}\right|}}{\sqrt{\left(\nu_{n}^{2}+(p-\mu)^{2}\right)\left(\nu_{n^{\prime}}^{2}+\left(p^{\prime}-\mu\right)^{2}\right)}}\right\} \theta\left(\hat{\delta}-\hat{\nu}_{n}\right) \theta\left(\hat{\delta}-\hat{\nu}_{n^{\prime}}\right) .
$$

for $n \neq n^{\prime}$ and

$$
K^{\circ}\left(n, p^{\prime} \mid n, p\right)=3\left\{\frac{\log \frac{p+p^{\prime}}{\left|p-p^{\prime}\right|}}{\sqrt{\left(\nu_{n}^{2}+(p-\mu)^{2}\right)\left(\nu_{n}^{2}+\left(p^{\prime}-\mu\right)^{2}\right)}}\right\} \theta\left(\hat{\delta}-\hat{\nu}_{n}\right) .
$$

To calculate the leading order behaviour we move to the continuum limit,

$$
\frac{1}{\beta} \sum_{n^{\prime}=0}^{\infty} \rightarrow \frac{1}{2 \pi} \int_{\frac{\epsilon}{2}}^{\infty} d \nu^{\prime}
$$

where $\epsilon=2 \pi k_{B} T$, and perform the momentum integral for $p^{\prime}$,

$$
\int_{p^{\prime} \approx \mu} d p^{\prime} \frac{1}{\left[\nu_{n^{\prime}}^{2}+\left(p^{\prime}-\mu\right)^{2}\right]} \rightarrow \frac{\pi}{\nu_{n^{\prime}}}
$$


(assuming $\nu_{n^{\prime}} \ll \mu$ ). Furthermore, the kernel $K^{\circ}$, containing both $-\log \left|\hat{\nu}-\hat{\nu}^{\prime}\right|$ and $-\log \mid \hat{\nu}+$ $\hat{\nu}^{\prime}$, can be treated using the same approximation as employed by Son [28]. This amounts to replacing the sum of these terms,

$$
\log \frac{1}{\left|\hat{\nu}-\hat{\nu}^{\prime}\right|}+\log \frac{1}{\left|\hat{\nu}+\hat{\nu}^{\prime}\right|} \approx 2 \log \frac{1}{\hat{\nu}_{>}}
$$

where $\hat{\nu}_{>}=\max \left(\hat{\nu}, \hat{\nu}^{\prime}\right)$. Although this approximation may appear drastic, in fact it captures the behaviour remarkably well. We will show in the next section that the correction to the full kernel due to this approximation does not contribute a linear logarithm but that the continuous-discrete energy approximation does.

In the continuum limit we incorporate (4.9), (4.10) and (4.11) into (4.6), and are left with the integral equation,

$$
\chi_{j}(\nu)=\lambda_{j}^{\circ 2} \frac{g^{2}}{24 \pi^{2}}\left(1+\frac{1}{N}\right) \int_{\frac{\hat{\epsilon}}{2}}^{\hat{\delta}} \frac{d \hat{\nu}^{\prime}}{\hat{\nu}^{\prime}} \log \frac{1}{\hat{\nu}_{>}} \chi_{j}\left(\nu^{\prime}\right),
$$

where the ultraviolet energy cut-off $\hat{\delta} \sim 1$ is analogous to the cut made in momentum for the toy model in (2.15). Note that the rescaling of $\nu$ in (4.4) results in a re-scaled infrared cutoff, $\hat{\epsilon}=\left(N_{f} / 2\right)^{5 / 2} \frac{g^{5} k_{B} T}{128 \pi^{3} \mu}$. While (4.12) may be solved by Son's method for arbitrary cutoff $\hat{\delta}$, the results take the simplest form when $\hat{\delta}=1$. In this case, we find the solutions

$$
\chi_{j}(\nu)=\theta(\hat{\delta}-\hat{\nu}) \frac{2}{\sqrt{\log 2 / \hat{\epsilon}}} \sin \left[\left(j+\frac{1}{2}\right) \pi \frac{\log 1 / \hat{\nu}}{\log 2 / \hat{\epsilon}}\right],
$$

with eigenvalues

$$
\frac{1}{\lambda_{j}^{\circ}}=\frac{g}{(2 j+1) \pi^{2}} \sqrt{\frac{N+1}{6 N}} \log \frac{2}{\hat{\epsilon}} \quad(\text { for } \hat{\delta}=1) .
$$

The functions $h_{j}(\nu, p)$ form a orthonormal set with respect to the continuum approximation, i.e.

$$
\int_{\frac{\epsilon}{2}}^{\delta} \frac{d \nu}{2 \pi} \int_{0}^{\infty} d p h_{i}(\nu, p) h_{j}(\nu, p)=\delta_{i j}
$$

where we have dropped the contribution from the lower limit of $p$ integration. This approximation will be adapted in the subsequent work.

For $\hat{\delta} \neq 1$, the eigenvalues are instead obtained by solution of the transcendental equation (6.2), resulting in

$$
\frac{1}{\lambda_{j}^{\circ}}=\frac{g}{(2 j+1) \pi^{2}} \sqrt{\frac{N+1}{6 N}}\left(\log \frac{2}{\hat{\epsilon}}+\alpha+\cdots\right),
$$

where

$$
\alpha=-\frac{1}{3}\left[\left(j+\frac{1}{2}\right) \pi\right]^{2} \frac{\log ^{3} 1 / \hat{\delta}}{\log ^{2} 2 / \hat{\epsilon}} .
$$


As may be seen from (4.9), the fermion Matsubara sum in the finite temperature formalism automatically provides a natural infrared cutoff to the continuum eigenvalue problem. Also, the dependence on the ultraviolet cutoff, $\hat{\delta}$, is beyond the order to which we are interested in, unlike in the toy model. This allows $\alpha$ to be ignored, so that (4.13) and (4.14) are sufficient in the subsequent calculations. More discussion on the dependence of the results on the ultraviolet cutoff is given in section VI.

One concern when defining the general eigenvalue problem is that, with a non-hermitian kernel (3.23), in general the smallest eigenvalue defined by (3.25) may be complex. Since the leading behavior in coupling of the transition temperature is solely determined by one gluon exchange, in analyzing this question we shall focus our attention on the corresponding eigenvalue problem with a kernel defined by (3.23) and (4.1). In this case, by direct calculation we have shown that the leading order of the eigenvalue is in fact real. We shall briefly show, further, that as long as it is not degenerate then the smallest eigenvalue must be real to all orders. To do so, we examine the characteristic polynomial of the eigenvalue problem, $\mathcal{P}(\rho)=\operatorname{det} P(\rho)$ where

$$
P_{s_{1}^{\prime} s_{2}^{\prime}, s_{1} s_{2}}(\rho) \equiv K_{s_{1}^{\prime} s_{2}^{\prime}, s_{1} s_{2}}-\rho \delta_{s_{1}^{\prime} s_{1}} \delta_{s_{2}^{\prime} s_{2}} .
$$

To investigate the coefficients of $\mathcal{P}(\rho)$ we first consider $\rho$ to be real. In this case, we find the relation

$$
P_{s_{1}^{\prime} s_{2}^{\prime}, s_{1} s_{2}}(\rho)=(-)^{\frac{s_{1}-s_{2}}{2}+\frac{s_{1}^{\prime}-s_{2}^{\prime}}{2}} P_{s_{2}^{\prime} s_{1}^{\prime}, s_{2} s_{1}}^{*}(\rho),
$$

by explicit examination of (3.23) for the one gluon exchange in different channels, tabulated in appendix A together with the property that

$$
S_{s_{1}}(n \mid p) S_{s_{2}}(-n \mid p)=S_{s_{2}}^{*}(n \mid p) S_{s_{1}}^{*}(-n \mid p)
$$

for a bare quark propagator. Therefore it follows that $\operatorname{det} P(\rho)=\operatorname{det} P^{*}(\rho)$, so that the coefficients of the characteristic polynomial are all real. Continuing $\rho$ to be complex, the real coefficients of the characteristic polynomial imply that the eigenvalues determined by the characteristic equation, $\mathcal{P}(\rho)=0$, are either real or appear in complex conjugate pairs. Therefore, if the smallest eigenvalue is not degenerate it is real. Although this argument is specifically for the problem defined by one gluon exchange, we expect similar reasoning to extend to the general eigenvalue problem.

\section{A. The Perturbation Series.}

Given the above leading order solution, we now turn to the issue of perturbative corrections. To simplify the notation, we remove non-essential factors by rescaling the kernel and eigenvalues by

$$
K_{S}^{J}\left(n^{\prime}, p^{\prime} \mid n, p\right)=\frac{g^{2}}{24 \pi^{2}}\left(1+\frac{1}{N}\right) \widetilde{K}_{S}^{J}\left(n^{\prime}, p^{\prime} \mid n, p\right),
$$

and 


$$
\frac{1}{\lambda_{j}^{2}}=\frac{g^{2}}{24 \pi^{2}}\left(1+\frac{1}{N}\right) \frac{1}{\widetilde{\lambda}_{j}^{2}} .
$$

Then to the leading order, we have $\widetilde{\lambda}_{0}=\widetilde{\lambda}_{0}^{\circ}$ with

$$
\frac{1}{\widetilde{\lambda}_{j}^{\circ}}=\frac{1}{\left(j+\frac{1}{2}\right) \pi} \log \frac{2}{\hat{\epsilon}} .
$$

One concern, that eigenvalues of the general problem may be complex, was discussed in the previous sub-section where it was found that the smallest eigenvalue - the eigenvalue of interest in defining the transition temperature - is real if it is not degenerate. Another concern in defining a perturbation series with discrete energy is that the leading order solutions $h_{j}^{\circ}\left(\nu_{n}, p\right)$ [obtained from the substitution $\nu \rightarrow \nu_{n}$ in (4.13)] are not orthogonal and cannot be directly used as a basis for the perturbative expansion. However, each of the solutions may be rotated slightly so that the resulting set forms a complete orthonormal basis. In a more convenient notation, in which $\left\langle\nu_{n}, p \mid j_{c}\right\rangle=h_{j}^{\circ}\left(\nu_{n}, p\right)$, while $\left\langle\nu_{n}, p \mid j\right\rangle=$ $\left\langle j_{c} \mid j_{c}\right\rangle^{-1 / 2} h_{j}^{\circ}\left(\nu_{n}, p\right)$ we can use Gram-Schmidt decomposition to write down an orthonormal basis,

$$
|\tilde{j}\rangle=\frac{|j\rangle-\sum_{i=0}^{j-1}|\tilde{i}\rangle\langle\tilde{i} \mid j\rangle}{\sqrt{1-\sum_{i=0}^{j-1}\langle j \mid \tilde{i}\rangle\langle\tilde{i} \mid j\rangle}}
$$

where all $|j\rangle$ are normalized with respect to the discrete sum over Matsubara energy, $|\tilde{0}\rangle=|0\rangle$ and other $|\tilde{j}\rangle$ for $j>0$ are found inductively. The norm $\left\langle j_{c} \mid j_{c}\right\rangle$ is examined in appendix B along with the inner product $\left\langle j_{c} \mid j_{c}^{\prime}\right\rangle$ and some matrix elements of the perturbative kernel. In this basis, we define the zeroth order operator with discrete energy,

$$
\mathcal{K}_{0}=\sum_{i} \tilde{\lambda}_{i}^{\circ-2}|\tilde{i}\rangle\langle\tilde{i}| .
$$

This operator has exactly the eigenvalues (4.14) by definition: $\mathcal{K}_{0}$ is diagonalized by $|\tilde{j}\rangle$ and so $|\tilde{j}\rangle=\tilde{\lambda}_{j}^{\circ} \mathcal{K}_{0}|\tilde{j}\rangle$. Thus $\mathcal{K}_{0}$ captures the desired leading order behavior of the full kernel at hand. In appendix $\mathrm{C}$ it is shown that in the continuum limit this operator in fact reduces exactly to the leading order kernel (4.7) that contains the double logarithmic behaviour.

We now write the full kernel of (3.27) in terms of this zeroth order operator and two correction terms,

$$
\widetilde{K}_{S}^{J=0}=\mathcal{K}_{0}+\mathcal{K}_{1}+\mathcal{K}_{2}
$$

where

$$
\mathcal{K}_{1}=K^{\circ}-\mathcal{K}_{0}
$$

contains both the effects of discrete to continuous energy corrections as well as corrections due to the approximation (4.11) and

$$
\mathcal{K}_{2}=\widetilde{K}_{S}^{J=0}-K^{\circ}
$$


accounts for corrections between $K^{\circ}$ and the full kernel $\widetilde{K}_{S}^{J=0}$. In particular, the subleading behaviour discarded by working in the continuum limit to derive (4.13) and (4.14) is contained in $\mathcal{K}_{1}$.

Following standard perturbation theory, the eigenvalue problem can be calculated up to second order, which proves sufficient to obtain all the next-to-leading order behaviour required. To define our perturbative expansion, we note that, to form a complete set, there are actually two types of wavefunction at zeroth order. The first type are determined by the kernel $K^{\circ}$ (and hence appear in the definition of $\mathcal{K}_{0}$ ), are denoted by $|\tilde{l}\rangle$ (which includes $|\tilde{0}\rangle$ ) and have eigenvalues $\tilde{\lambda}_{l}^{\circ-2}$. These wavefunctions are constrained to have support only in the domain of $K^{\circ}$ (i.e. $\left.\hat{\nu} \in[\hat{\epsilon} / 2, \hat{\delta}]\right)$, and are zero outside. The second type of wavefunction are not yet determined; to distinguish them from the first type they are denoted by $\mid m)$ and have eigenvalues $\widetilde{\lambda}_{m}^{-2}=0$. Together, these solutions give us the completeness relation,

$$
1=|0\rangle\left\langle 0\left|+\sum_{j \neq 0}\right| \tilde{j}\right\rangle\left\langle\tilde{j}\left|+\sum_{m}\right| m\right)(m \mid
$$

Using second order perturbation theory and the completeness relation, we find

$$
\begin{aligned}
& \frac{1}{\widetilde{\lambda}_{0}^{2}}=\frac{1}{\widetilde{\lambda}_{0}^{\circ}}+\left\langle\tilde{0}\left|\left(\mathcal{K}_{1}+\mathcal{K}_{2}\right)\right| \tilde{0}\right\rangle
\end{aligned}
$$

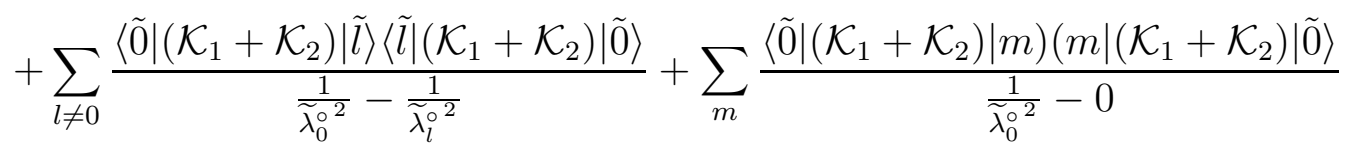

$$
\begin{aligned}
& =\frac{1}{\widetilde{\lambda}_{0}^{\circ 2}}+\left\langle\tilde{0}\left|\left(\mathcal{K}_{1}+\mathcal{K}_{2}\right)\right| \tilde{0}\right\rangle+\widetilde{\lambda}_{0}^{\circ}\left\langle\tilde{0}\left|\left(\mathcal{K}_{1}+\mathcal{K}_{2}\right)^{2}\right| \tilde{0}\right\rangle-\tilde{\lambda}_{0}^{\circ^{2}}\left\langle\tilde{0}\left|\left(\mathcal{K}_{1}+\mathcal{K}_{2}\right)\right| \tilde{0}\right\rangle^{2} \\
& +\sum_{l \neq 0}\left(\frac{1}{\frac{1}{{\tilde{\lambda_{0}^{\circ}}}^{2}}-\frac{1}{\tilde{\lambda}_{l}^{\circ}}}-\tilde{\lambda}_{0}^{\circ}\right)\left\langle\tilde{0}\left|\left(\mathcal{K}_{1}+\mathcal{K}_{2}\right)\right| \tilde{l}\right\rangle\left\langle\tilde{l}\left|\left(\mathcal{K}_{1}+\mathcal{K}_{2}\right)\right| \tilde{0}\right\rangle
\end{aligned}
$$

which may be compared to the analogous expression for the toy model, (2.20).

We now proceed to evaluate (4.30) and to resolve the eigenvalue problem to next-toleading order in the logarithmic behaviour. This will not only determine the exponential and the pre-exponential factor of the transition temperature to the superphase of QCD to leading order in the weak coupling regime but also allow us to identify the physical origins of the contributions. Since the leading order is double logarithmic, the sub-leading constant pertaining to the logarithm is solely determined by linearly logarithmic terms. Contributions of $\mathcal{O}(1)$ may be dropped.

\section{B. First order perturbation.}

The first order term in (4.30) is evaluated in two parts, the first of which is the expectation of $\mathcal{K}_{1}$. This itself can be separated into two parts: $\mathcal{K}_{1}^{(a)}$ containing corrections due to the approximation of the log terms given in (4.11), and $\mathcal{K}_{1}^{(b)}$ containing significant continuousdiscrete energy corrections, 


$$
\begin{gathered}
\mathcal{K}_{1}=\mathcal{K}_{1}^{(a)}+\mathcal{K}_{1}^{(b)}, \\
\mathcal{K}_{1}^{(a)}=\theta\left(\hat{\delta}-\hat{\nu}_{n}\right) \theta\left(\hat{\delta}-\hat{\nu}_{n^{\prime}}\right)\left[\frac{\log \frac{1}{\left|\hat{\nu}_{n}-\hat{\nu}_{n^{\prime}}\right|}+\log \frac{1}{\left|\hat{\nu}_{n}+\hat{\nu}_{n^{\prime}}\right|}-2 \log \frac{1}{\hat{\nu}_{>}}}{\sqrt{\left(\nu_{n}^{2}+(p-\mu)^{2}\right)\left(\nu_{n^{\prime}}^{2}+\left(p^{\prime}-\mu\right)^{2}\right)}}\left(1-\delta_{n n^{\prime}}\right)\right. \\
\left.+\frac{3 \log \frac{p+p^{\prime}}{\left|p-p^{\prime}\right|}-2 \log \frac{1}{\hat{\nu}>}}{\sqrt{\left(\nu_{n}^{2}+(p-\mu)^{2}\right)\left(\nu_{n^{\prime}}^{2}+\left(p^{\prime}-\mu\right)^{2}\right)}} \delta_{n n^{\prime}}\right], \\
\mathcal{K}_{1}^{(b)}=\theta\left(\hat{\delta}-\hat{\nu}_{n}\right) \theta\left(\hat{\delta}-\hat{\nu}_{n^{\prime}}\right) \frac{1}{\hat{\nu}_{>}}-\mathcal{K}_{0} .
\end{gathered}
$$

In appendix $\mathrm{D}$ it is shown that the contribution from $\mathcal{K}_{1}^{(a)}$ may be ignored,

$$
\left\langle\tilde{0}\left|\mathcal{K}_{1}^{(a)}\right| \tilde{0}\right\rangle=\mathcal{O}(1)
$$

Significant continuous-discrete energy corrections do, however, arise from the expectation of $\mathcal{K}_{1}^{(b)}$. This expression is evaluated using Zeta function techniques, and details of the calculation are given in appendix B. In total, we find the contribution from the first term to be,

$$
\left\langle\tilde{0}\left|\mathcal{K}_{1}\right| \tilde{0}\right\rangle=\frac{4}{\pi^{2}}\left[2(\gamma+\log 2) \log \frac{2}{\hat{\epsilon}}+\mathcal{O}(1)\right]
$$

The second term to be evaluated at first order, $\mathcal{K}_{2}$, contains the correction between the full kernel for one gluon exchange and the approximate kernel containing $K^{\circ}$. The first order perturbation of $\mathcal{K}_{2}$ is given by,

$$
\begin{aligned}
\left\langle\tilde{0}\left|\mathcal{K}_{2}\right| \tilde{0}\right\rangle & =\frac{1}{\beta^{2}} \sum_{n, n^{\prime}} \int_{0}^{\infty} d p \int_{0}^{\infty} d p^{\prime} \frac{\widetilde{K}_{S}^{J=0}\left(n^{\prime}, p^{\prime} \mid n, p\right)-K^{\circ}\left(n^{\prime}, p^{\prime} \mid n, p\right)}{\sqrt{\left(\nu_{n}^{2}+(p-\mu)^{2}\right)\left(\nu_{n^{\prime}}^{2}+\left(p^{\prime}-\mu\right)^{2}\right)}} \chi_{0}\left(\nu_{n}\right) \chi_{0}\left(\nu_{n^{\prime}}\right) \\
& =\int_{\frac{\epsilon}{2}}^{\delta} \frac{d \nu}{2 \pi} \int_{\frac{\epsilon}{2}}^{\delta} \frac{d \nu^{\prime}}{2 \pi} \chi_{0}(\nu) \chi_{0}\left(\nu^{\prime}\right) \int_{0}^{\infty} d p \int_{0}^{\infty} d p^{\prime} \frac{\widetilde{K}_{S}^{J}-K^{\circ}}{\sqrt{\left(\nu^{2}+(p-\mu)^{2}\right)\left(\nu^{\prime 2}+\left(p^{\prime}-\mu\right)^{2}\right)}}
\end{aligned}
$$

To produce a linear logarithmic term, or in other words to knock out one logarithmic power relative to the leading order, both $\nu$ and $\nu^{\prime}$ should be $\sim k_{B} T$. If either $\nu$ or $\nu^{\prime}$ becomes large in comparison with $k_{B} T$ it will bring the integrand away from the corresponding Fermi sea and therefore away from the sensitive region of the gluon propagators so two powers of a logarithm will be eliminated. For this reason, the integrations over $p$ and $p^{\prime}$ are dominated at the poles $p-\mu= \pm i \nu$ and $p^{\prime}-\mu= \pm i \nu^{\prime}$ and the energy transfer square in the denominator of the magnetic gluon propagator can be disregarded.

If the behaviour of (4.36) is in fact linearly logarithmic it would be tied to the constant pertaining to the logarithms of $K^{\circ}$. Thus (4.36) demands a closer look and we start with the partial wave integrals of single electric and magnetic gluon interaction that combine to give $\tilde{\gamma}^{J=0}$,

$$
\tilde{\gamma}^{J=0}=-\frac{g^{2}}{2}\left(1+\frac{1}{N}\right)\left[\tilde{\gamma}^{(M)}+\tilde{\gamma}^{(E)}\right]
$$


In the case $p=p^{\prime}=\mu$,

$$
\tilde{\gamma}^{(M)} \simeq \frac{1}{4} \int_{-1}^{1} d \cos \theta \frac{3-\cos \theta}{2 \mu^{2}(1-\cos \theta)+m_{D}^{2} f_{M}(x)}=2 \int_{0}^{1} d \xi \xi \frac{1+\xi^{2}}{4 \mu^{2} \xi^{2}+m_{D}^{2} f_{M}\left(\frac{\hat{\omega}}{2 \xi}\right)}
$$

and

$$
\tilde{\gamma}^{(E)} \simeq \frac{1}{4} \int_{-1}^{1} d \cos \theta \frac{1+\cos \theta}{2 \mu^{2}(1-\cos \theta)+m_{D}^{2} f_{E}(x)}=2 \int_{0}^{1} d \xi \xi \frac{1-\xi^{2}}{4 \mu^{2} \xi^{2}+m_{D}^{2} f_{E}\left(\frac{\hat{\omega}}{2 \xi}\right)}
$$

where $\hat{\omega}=\omega / \mu$ and we have changed the integration variable from $\cos \theta$ to $\xi=\sin \frac{\theta}{2}$. We first note that the $\xi^{2}$ terms in the numerator are free from the collinear singularity. For these parts the self-energy functions in the denominators can be dropped and we obtain by trivial integration a constant $\frac{1}{4 \mu^{2}}$ from $\mathcal{K}_{2}^{(M)}$ and a constant $-\frac{1}{4 \mu^{2}}$ from $\mathcal{K}_{2}^{(E)}$. Amazingly, these sub-leading contributions of the electric and magnetic parts exactly cancel in (4.36). As we shall in section $\mathrm{V}$, this is not the case with $J \neq 0$.

We now focus on the more difficult infrared sensitive parts of the above integrals, namely

$$
I_{M}=\int_{0}^{1} d \xi \frac{\xi}{4 \mu^{2} \xi^{2}+m_{D}^{2} f_{M}\left(\frac{\hat{\omega}}{2 \xi}\right)}
$$

and,

$$
I_{E}=\int_{0}^{1} d \xi \frac{\xi}{4 \mu^{2} \xi^{2}+m_{D}^{2} f_{E}\left(\frac{\hat{\omega}}{2 \xi}\right)} .
$$

For the first integral, we introduce $\xi_{0}$ such that $\hat{\omega} \ll \xi_{0} \ll(\eta \hat{\omega})^{\frac{1}{3}}$ with $\eta=\frac{\pi m_{D}^{2}}{32 \mu^{2}}$ and divide the integration domain $0<\xi<1$ into the two regions; $0<\xi<\xi_{0}$ and $\xi_{0}<\xi<1$. Correspondingly, we have $I_{M}=I_{M}^{>}+I_{M}^{<}$with,

$$
\begin{aligned}
& I_{M}^{>}=\int_{\xi_{0}}^{1} d \xi \frac{\xi}{4 \mu^{2} \xi^{2}+m_{D}^{2} f_{M}\left(\frac{\hat{\omega}}{2 \xi}\right)}, \\
& I_{M}^{<}=\int_{0}^{\xi_{0}} d \xi \frac{\xi}{4 \mu^{2} \xi^{2}+m_{D}^{2} f_{M}\left(\frac{\hat{\omega}}{2 \xi}\right)} .
\end{aligned}
$$

The Landau damping approximation can then be applied to $\sigma^{M}$ in (4.42) and the integration gives rise to the leading order logarithm included in $K^{\circ}$ without any additional constant. As for (4.43), on the other hand, the mean-value theorem implies that,

$$
I_{M}^{<}=\xi_{0} \frac{\bar{\xi}}{4 \mu^{2} \bar{\xi}^{2}+m_{D}^{2} f_{M}\left(\frac{\hat{\omega}}{2 \bar{\xi}}\right)}
$$

for some $\bar{\xi}$ in the region $0<\bar{\xi}<\xi_{0}$. Since $f_{M}(x)$ is a monotonically increasing function of $x$, we find, 


$$
I_{M}^{<} \leq \xi_{0} \frac{\bar{\xi}}{m_{D}^{2} f_{M}\left(\frac{\hat{\omega}}{2 \bar{\xi}}\right)} \leq \frac{\xi_{0}^{2}}{m_{D}^{2} f_{M}\left(\frac{\hat{\omega}}{2 \xi_{0}}\right)} \simeq \frac{\xi_{0}^{3}}{\eta \hat{\omega}} \ll 1 .
$$

Therefore no additional constant is found from $I_{M}$ either.

The integral $I_{E}$ is more subtle than $I_{M}$ because of the dielectric behavior at high $\omega$. In this case we have to introduce two cutoffs, $\xi_{0}$ and $\xi_{0}^{\prime}$ such that $\hat{\omega} \xi_{0} \ll \xi_{0}^{\prime} \ll \hat{\omega} \ll \xi_{0} \ll 1$, and write $I_{E}=I_{E}^{(a)}+I_{E}^{(b)}+I_{E}^{(c)}$, where,

$$
\begin{aligned}
& I_{E}^{(a)}=\int_{\xi_{0}}^{1} d \xi \frac{\xi}{4 \mu^{2} \xi^{2}+m_{D}^{2} f_{E}\left(\frac{\hat{\omega}}{2 \xi}\right)}, \\
& I_{E}^{(b)}=\int_{\xi_{0}^{\prime}}^{\xi_{0}} d \xi \frac{\xi}{4 \mu^{2} \xi^{2}+m_{D}^{2} f_{E}\left(\frac{\hat{\omega}}{2 \xi}\right)},
\end{aligned}
$$

and

$$
I_{E}^{(c)}=\int_{0}^{\xi_{0}^{\prime}} d \xi \frac{\xi}{4 \mu^{2} \xi^{2}+m_{D}^{2} f_{E}\left(\frac{\hat{\omega}}{2 \xi}\right)} .
$$

The integral $I_{E}^{(a)}$ gives rise to the logarithm included in $K^{\circ}$ without any additional constant. The mean-value theorem, applied to $I_{E}^{(b)}$ implies, with $\xi_{0}^{\prime}<\bar{\xi}<\xi_{0}$, that,

$$
I_{E}^{(b)}=\frac{\left(\xi_{0}-\xi_{0}^{\prime}\right) \bar{\xi}}{4 \mu^{2} \bar{\xi}^{2}+m_{D}^{2} f_{E}\left(\frac{\hat{\omega}}{2 \bar{\xi}}\right)} \leq \frac{\xi_{0}^{2}}{m_{D}^{2} f_{E}\left(\frac{\hat{\omega}}{2 \xi_{0}^{\prime}}\right)} \simeq \frac{\xi_{0}^{2} \hat{\omega}^{2}}{\xi_{0}^{\prime 2}} \ll 1 .
$$

Finally, the integral $I_{E}^{(c)}$ is divergent logarithmically at the lower limit. However this is an artifact of setting $p=p^{\prime}=\mu$. Without this approximation, one has to consider the partial wave integration simultaneously with the integrations of $p$ and $p^{\prime}$. The net result, with $p-p^{\prime} \sim \omega$, amounts to a cutoff $\xi \sim \hat{\omega}^{4}$ and we expect,

$$
I_{E}^{(c)} \sim \frac{1}{4 \mu^{2}} \hat{\omega}^{2} \log \frac{1}{\hat{\omega}}
$$

which is completely infrared safe. Thus we have demonstrated that there is no constant from $\mathcal{K}_{2}^{(M)}+\mathcal{K}_{2}^{(E)}$ in addition to the leading logarithms in the limit $\omega \rightarrow 0$. As the result the first order perturbation (4.36) leads to no additional linear log contributions.

\section{Second order perturbation.}

Now we proceed to the last three terms of (4.30), which stand for the second order corrections in $\mathcal{K}_{1}+\mathcal{K}_{2}$. Firstly, the penultimate term of (4.30) is easy to dispose of; although

$\left\langle\tilde{0}\left|\left(\mathcal{K}_{1}+\mathcal{K}_{2}\right)\right| \tilde{0}\right\rangle^{2} \sim \log ^{2} \frac{2}{\hat{\epsilon}}$ this is suppressed by the factor ${\lambda_{0}^{\circ}}^{2} \sim\left(\log \frac{2}{\hat{\epsilon}}\right)^{-2}$, so that it would contribute at most at the constant level, and not at the linear log level of interest.

Secondly we consider the final term of (4.30), containing the sum over discrete intermediate states. For this term, the arguments given above at first order also enable us the 
disregard $\mathcal{K}_{2}$ when it is sandwiched between $|\tilde{0}\rangle$ and $|\tilde{l}\rangle$. As a result, we only need to consider $\left\langle\tilde{0}\left|\mathcal{K}_{1}\right| \tilde{l}\right\rangle$ which is at most linear in logarithms. Therefore the same power counting argument used for the penultimate term also applies here. There is no linear logarithm associated with the last term and it can be dropped.

Finally, the remaining term involves the second order matrix element

$$
\left\langle\tilde{0}\left|\left(\mathcal{K}_{1}+\mathcal{K}_{2}\right)^{2}\right| \tilde{0}\right\rangle=I_{\text {in }}+I_{\text {out }},
$$

where,

$$
\begin{aligned}
I_{\text {in }} \simeq & \frac{1}{\beta^{3}} \sum_{\substack{n, m, n^{\prime} \\
\nu m<\delta}} \chi_{0}\left(\nu_{n}\right) \chi_{0}\left(\nu_{n^{\prime}}\right) \int_{0}^{\infty} d p \int_{0}^{\infty} d q \int_{0}^{\infty} d p^{\prime} \\
& \times \frac{\left[\mathcal{K}_{1}(n, p \mid m, q)+\mathcal{K}_{2}(n, p \mid m, q)\right]\left[\mathcal{K}_{1}\left(m, q \mid n^{\prime}, p^{\prime}\right)+\mathcal{K}_{2}\left(m, q \mid n^{\prime}, p^{\prime}\right)\right]}{\sqrt{\left[\nu_{n}^{2}+(p-\mu)^{2}\right]\left[\nu_{n^{\prime}}^{2}+\left(p^{\prime}-\mu\right)^{2}\right]}},
\end{aligned}
$$

and

$$
\begin{aligned}
& I_{\mathrm{out}} \simeq \frac{1}{\beta^{3}} \sum_{\substack{n, m, n^{\prime} \\
\nu_{m}>\delta}} \chi_{0}\left(\nu_{n}\right) \chi_{0}\left(\nu_{n^{\prime}}\right) \int_{0}^{\infty} d p \int_{0}^{\infty} d q \int_{0}^{\infty} d p^{\prime} \\
& \times \frac{\left[\tilde{\gamma}^{J=0}(n, p \mid m, q)+\tilde{\gamma}^{J=0}(n, p \mid-m, q)\right]\left[\tilde{\gamma}^{J=0}\left(m, q \mid n^{\prime}, p^{\prime}\right)+\tilde{\gamma}^{J=0}\left(-m, q \mid n^{\prime}, p^{\prime}\right)\right]}{\left[\nu_{n}^{2}+(p-\mu)^{2}\right]\left[\nu_{m}^{2}+(q-\mu)^{2}\right]\left[\nu_{n^{\prime}}^{2}+\left(p^{\prime}-\mu\right)^{2}\right]} \\
& \simeq \frac{1}{4} \int_{\frac{\hat{\epsilon}}{2}}^{\hat{\delta}} \frac{d \hat{\nu}}{\hat{\nu}} \int_{\frac{\hat{\epsilon}}{2}}^{\hat{\delta}} \frac{d \hat{\nu}^{\prime}}{\hat{\nu}^{\prime}} \chi_{0}(\nu) \chi_{0}\left(\nu^{\prime}\right) \int_{\delta}^{\infty} d \nu^{\prime \prime} \int_{0}^{\infty} d q \\
& \times \frac{\left[\log \frac{1}{\left|\nu-\nu^{\prime \prime}\right|}+\log \frac{1}{\nu+\nu^{\prime \prime}}\right]\left[\log \frac{1}{\left|\nu^{\prime \prime}-\nu^{\prime}\right|}+\log \frac{1}{\nu^{\prime \prime}+\nu^{\prime}}\right]}{\nu^{\prime \prime 2}+(q-\mu)^{2}} .
\end{aligned}
$$

Consider $I_{\text {in }}$ first. The leading logarithm of the magnetic gluon propagator has been subtracted in $\mathcal{K}_{1}$ and we may regard $\mathcal{K}_{1}$ as bounded in magnitude. The most dangerous contribution comes from the pole term of the integrations over $p, p^{\prime}$ and $q$. The summations over $n, m$ and $n^{\prime}$ give rise to a contribution of order at most $\log ^{2} \frac{2}{\hat{\epsilon}}$ (taking into account the inverse square root of $\log \frac{2}{\hat{\epsilon}}$ from the normalization constant of each $\chi_{0}$ ). The net power of logarithms, when multiplied by $\tilde{\lambda}^{\circ}$ is thus zero. As to $I_{\text {out }}$, since the intermediate energy $\nu^{\prime \prime}$ is kept away from the Fermi level, the logarithms in the numerator of (4.53) are smeared. We are left with only two powers of logarithms from the lower limits of the $\nu$ and $\nu^{\prime}$ integrations and one inverse power of logarithm from the normalization constant of $\chi_{0}(\nu)$. The net power of logarithms, when multiplied by $\tilde{\lambda}^{{ }^{2}}$, is -1 . Therefore we do not find any contribution to the linear logarithmic term from second order perturbation theory.

In fact the only non-vanishing contribution at the linear log level, (4.35), is due to the discrete to continuous energy correction, and is obtained at first order in the perturbative expansion. Putting these results together, the expansion of the inverse square of the largest eigenvalue associated to the Fredholm equation with free quark propagators reads

$$
\frac{1}{\widetilde{\lambda}_{0}^{2}}=\frac{1}{\widetilde{\lambda}_{0}^{\circ}}+\frac{8}{\pi^{2}}(\gamma+\log 2) \log \frac{2}{\hat{\epsilon}}+\mathcal{O}(1)
$$


The linear logarithmic term of (4.54) contributes to the factor $c_{1}^{\prime \prime}$ of (1.6). Our analytical arguments on the perturbation corrections are confirmed by numerical integration, as will be discussed in section VI.

\section{NON-ZERO ANGULAR MOMENTUM.}

Although we have focused on the $s$-wave channel for longitudinal pairs of quarks, the collinear singularity of one-gluon exchange which is responsible for the long-range nature

of the pairing interaction makes no distinction at leading order between zero and non-zero angular momenta. Therefore, in any angular momentum $J$ channel, the largest factor contributing to the critical temperature, $k_{B} T_{C}^{J}$, is given by the non-BCS relation (1.3) in all cases. However, the constant pertaining to the logarithm may vary with angular momentum. For the $J^{\text {th }}$ partial wave, the increasing number of nodes of the Legendre polynomial $P_{J}(\cos \theta)$ with increasing $J$ reduces the effective attraction between quarks. As we shall see, although formally of $\mathcal{O}(1)$, the suppression for $J \neq 0$ can be of several orders of magnitude.

To explore such a correlation, we evaluate the $J \neq 0$ partial wave component of the irreducible vertex function,

$$
\begin{aligned}
\tilde{\gamma}^{J}\left(n^{\prime}, p^{\prime} \mid n, p\right)= & -\frac{g^{2}}{8}\left(1+\frac{1}{N}\right)(2 J+1) \int_{-1}^{1} d(\cos \theta) P_{J}(\cos \theta) \\
& \times\left[\frac{3-\cos \theta-(1+\cos \theta) \frac{\left(p-p^{\prime}\right)^{2}}{\left|\vec{p}-\vec{p}^{\prime}\right|^{2}}}{\left(\nu_{n}-\nu_{n^{\prime}}\right)^{2}+\left|\vec{p}-\vec{p}^{\prime}\right|^{2}+m_{D}^{2} f_{M}(x)}+\frac{1+\cos \theta}{\left|\vec{p}-\vec{p}^{\prime}\right|^{2}+m_{D}^{2} f_{E}(x)}\right] .
\end{aligned}
$$

To uncover the dependence on $J$, we set $p=p^{\prime}=\mu$. The numerator of the integrand can be decomposed into a constant term and a non-constant term with the latter vanishing in the forward direction, $\cos \theta=1$. Since $P_{J}(1)=1$ for any $J$, the contribution from the constant term is independent of $J$ and can be approximated by the $J=0$ vertex given by Eqn. (4.2). For the non-constant part of the numerator, proportional to $1-\cos \theta$, the forward direction is infrared safe, and the self energies $f_{M}$ and $f_{E}$ may be dropped to the order we are interested in. The resultant integral produces a sub-leading $J$-dependent term. We find,

$$
\tilde{\gamma}^{J}\left(n^{\prime}, p^{\prime} \mid n, p\right)=-\frac{g^{2}}{12 \mu^{2}}\left(1+\frac{1}{N}\right)(2 J+1)\left(\log \frac{1}{\left|\hat{\nu}_{n}-\hat{\nu}_{n^{\prime}}\right|}+3 c_{J}\right)
$$

with,

$$
c_{J}=\int_{-1}^{1} d \cos \theta \frac{P_{J}(\cos \theta)-1}{1-\cos \theta}
$$

Using the recursion formula for Legendre polynomials,

$$
(J+1) P_{J+1}(\cos \theta)-(2 J+1) \cos \theta P_{J}(\cos \theta)+J P_{J-1}(\cos \theta)=0,
$$

we find that,

$$
(J+1) c_{J+1}-(2 J+1) c_{J}+J c_{J-1}=-2 \delta_{J 0} .
$$


Since $c_{0}=0$, the solution to (5.5) for $J \geq 1$ is,

$$
c_{J}=-2 \sum_{n=1}^{J} \frac{1}{n} .
$$

Following the arguments of the perturbation theory for $J=0$, we see that $c_{J}$ contributes only to the first order perturbation of the eigenvalue, and hence the critical temperature in the angular momentum $J$ channel reads,

$$
\begin{aligned}
k_{B} T_{C}^{J \neq 0} & =e^{3 c_{J}} k_{B} T_{C}^{J=0} \\
& \simeq 2.5 \times 10^{-3} k_{B} T_{C}^{J=0} \quad \text { for } J=1,
\end{aligned}
$$

where $T_{C}^{J=0}$ is the corresponding critical temperature in the $J=0$ channel.

The foregoing consideration can be generalized to transverse pairings between opposite helicities. The only modifications include replacing $3-\cos \theta$ in (5.1) by $1+\cos \theta$ and $P_{J}(\cos \theta)$ by $d_{11}^{J}(\theta)$, the Wigner D-functions [36]. The final formula for the transition temperature is given by (5.7) with $c_{J}$ replaced by

$$
\frac{1}{2}\left(c_{J}+\frac{J}{2 J+1} c_{J+1}+\frac{J+1}{2 J+1} c_{J-1}\right)
$$

with $J \geq 1$ and $c_{0}=0$. Relative to the longitudinal pairing at $J=0$, we find that for $J=1$ a longitudinal pairing is suppressed by a factor of $e^{-6}$ whereas a transverse pairing is suppressed by $e^{-4.5}$.

Physically, (5.7) shows a large suppression of the transition temperature with non-zero angular momentum relative to that for $s$-wave pairing. As the quark-gluon plasma is cooled from the normal phase, the first critical temperature reached is $T_{C}^{J=0}$ and the corresponding long range order is then switched on. This would then invalidate the relation (5.7) for $J \neq 0$, which was derived using normal phase propagators. Furthermore, there is no simple relation between $T_{C}^{J \neq 0}$ and the corresponding gap energy at $T=0$. Nevertheless, the suppression in (5.7) indicates that if for some physical reasons the long range order with $J=0$ cannot be formed, then the $J \neq 0$ pairing can only happen at very low temperatures.

\section{NUMERICAL RESULTS AND GAUGE INVARIANCE}

In the previous section we have examined the perturbative expansion of the full eigenvalue problem, (3.28), and have found a single correction term at the linear log level, arising from the discrete to continuum energy approximation, (4.35). While this result is simply stated, it came from an involved perturbative treatment of the Fredholm integral equation to second order. Thus, as a check, the analytical arguments on the perturbation corrections have been confirmed by numerical evaluation of both the first and second order terms in the perturbative expansion, (4.30).

At this point, a comment on the integration limits is in order. We recall that a cutoff, $\hat{\nu}<\hat{\delta}$, was imposed in order to control the zeroth order kernel, (4.7). As this cutoff is not otherwise physical, its dependence ought to drop out of the final result. This in fact has been demonstrated in the toy model of section II, where the cutoff dependence cancels between 
the zeroth and first order terms. For the actual case at hand, however, the cutoff dependence behaves differently. Consider the zeroth order one-gluon exchange kernel, (4.12), which may be rewritten in terms of the variable $x=\log \hat{\nu}$ :

$$
f(x)=-\widetilde{\lambda}^{2} \int_{a}^{b} d x^{\prime} x_{>} f\left(x^{\prime}\right),
$$

where $a=\log \frac{\hat{\epsilon}}{2}$ and $b=\log \hat{\delta}$. The exact eigenvalues of (6.1) may be obtained using Son's method [28]. The resulting spectrum is given by the zeros of the transcendental equation,

$$
b \widetilde{\lambda}=-\cot \tilde{\lambda}(b-a),
$$

and may be developed perturbatively for $(b / a) \ll 1$. We find,

$$
\frac{1}{\widetilde{\lambda}_{j}^{2}}=\left[\left(j+\frac{1}{2}\right) \pi\right]^{-2}\left(\log \frac{2}{\hat{\epsilon}}\right)^{2}\left[1+\frac{2}{3}\left[\left(j+\frac{1}{2}\right) \pi\right]^{2}\left(\frac{\log \hat{\delta}}{\log \frac{2}{\hat{\epsilon}}}\right)^{3}+O\left(\frac{\log \hat{\delta}}{\log \frac{2}{\hat{\epsilon}}}\right)^{5}\right],
$$

which corresponds to Eqn. (4.16) for $\hat{\delta} \neq 1$. This indicates that the cutoff dependence of the zeroth order term is in fact down by three powers of the logarithm, and hence contributes below the level we are interested in. This in fact explains why, unlike the toy model, we find no cutoff dependence at the linear log level term by term in the perturbative expansion (4.30). Thus our numerical work for the above was performed with a fixed upper cutoff of $\hat{\delta}=1$.

In addition to numerical confirmation of the perturbative expansion, we have also investigated the numerical solution to the Fredholm equation (3.28). This provides both a direct verification of our analytic results and a preliminary demonstration of the gauge invariance of our approach. Working in the normal phase makes treatment of gauge invariance easier to handle. In particular, as discussed in [31], the Fredholm determinant can be represented as a series of bubble diagrams, the sum of which is gauge invariant.

As this numerical work is secondary to the analytical results presented above, we make several simplifying approximations. Firstly, to avoid infinite sums, we only work with a continuous energy integral. As a consequence, we will not reproduce the discrete to continuum energy correction of Eqn. (4.35). Secondly, we a priori dispose of the momentum integral by noting that the dominant contribution to the kernel occurs near the Fermi surface, $\left\{p, p^{\prime}\right\} \approx \mu$. These two approximations correspond to the steps taken in Eqns. (4.9) and (4.10) in deriving the zeroth order integral equation, (4.12). Numerically, however, we do not employ Son's approximation, (4.11), and instead use the complete one gluon exchange vertex (evaluated with momenta at the Fermi surface).

One difficulty immediately arises as a consequence of setting $p=p^{\prime}=\mu$ in the one gluon exchange kernel, and that is the spurious divergence in the Coulomb propagator, (3.9), in the forward direction. While this divergence is eliminated by a full treatment incorporating momenta off of the Fermi surface, as shown in (4.50), here we have no such luxury without reintroducing the momentum integral. This difficulty prompts us to work in the covariant gauge, using the propagators of [29]. Using the covariant gauge propagators directly in numerical analysis of the integral equation provides a first check upon the gauge invariance of the approach. 


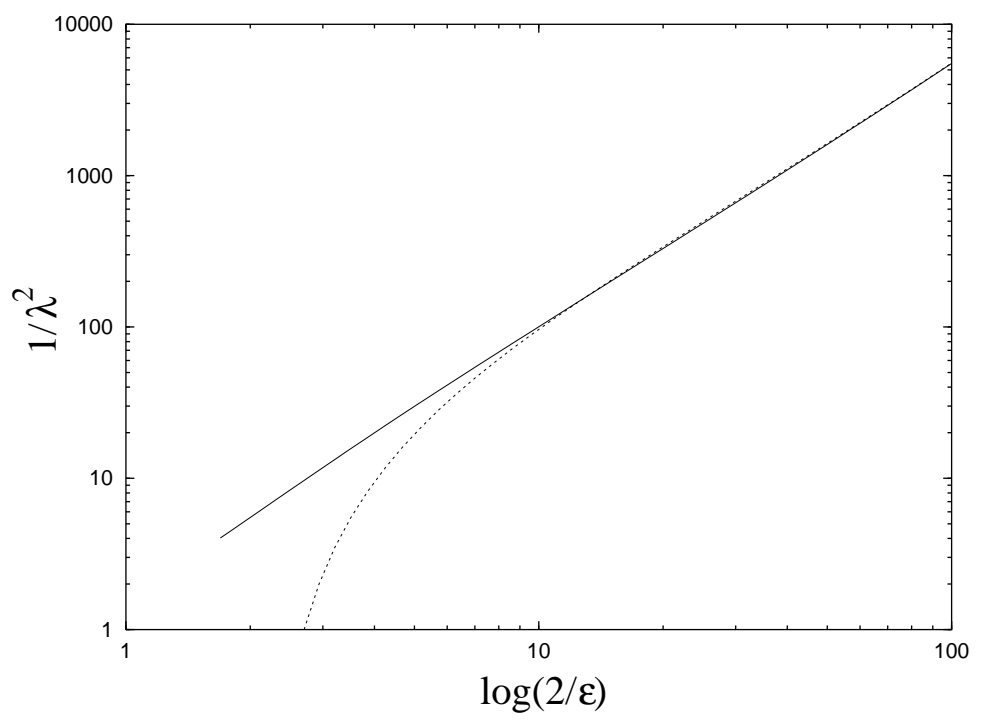

FIG. 3. The largest eigenvalue, $1 / \widetilde{\lambda}^{2}$, of the Fredholm problem $(6.4)$ as a function of $\log \frac{2}{\epsilon}$. The numerical solution is given by the solid line, while the zeroth order solution, (6.7), is given by the dashed line.

Taking everything into consideration, we thus examine the numerical solution to the integral equation,

$$
\chi(\nu)=\widetilde{\lambda}^{2} \int_{\frac{\epsilon}{2}}^{\delta} \frac{d \nu^{\prime}}{\nu^{\prime}} \widetilde{K}\left(\nu, \nu^{\prime}\right) \chi\left(\nu^{\prime}\right)
$$

with kernel

$$
\widetilde{K}\left(\nu, \nu^{\prime}\right)=3\left[\gamma\left(\nu, \nu^{\prime}\right)+\gamma\left(\nu,-\nu^{\prime}\right)\right]
$$

For the one gluon exchange interaction in covariant gauge, we use the result of [29]. Namely, in our notation,

$\gamma\left(\nu, \nu^{\prime}\right)=2 \int_{0}^{1} d \xi \xi\left[\frac{1+\xi^{2}}{\left(\nu-\nu^{\prime}\right)^{2}+4 \mu^{2} \xi^{2}+m_{D}^{2} f_{M}(x)}+\frac{1-\xi^{2}}{\left(\nu-\nu^{\prime}\right)^{2}+4 \mu^{2} \xi^{2}+m_{D}^{2}\left(1+x^{2}\right) f_{E}(x)}\right]$,

where $x=\left|\nu-\nu^{\prime}\right| /(2 \mu \xi)$. Note that the electric gluon exchange term is modified in covariant gauge, as may be seen by comparison with (4.38) and (4.39). For this linear Fredholm problem, which is similar to the non-linear gap equation, the numerical solution for the largest eigenvalue is easily obtained by iteration. One subtlety arises in this case, however, in that the relation between $T_{C}$ and the gauge coupling $g$ is given by inversion of the critical temperature condition, $\lambda^{2}\left(T_{c}, g, \mu\right)=1$. To investigate the behavior of (6.4) near the critical point, we thus use the zeroth order relation, $g^{2}\left(1+\frac{1}{N}\right)=6 \pi^{4}\left(\log \frac{2}{\epsilon}\right)^{-2}$, where $\epsilon=2 \pi k_{B} T_{C}$, to fix $m_{D}^{2}$ in (6.6). For explicit numerical work, we furthermore set $\delta=10^{10} \mu$, thus employing a finite but large cutoff. 


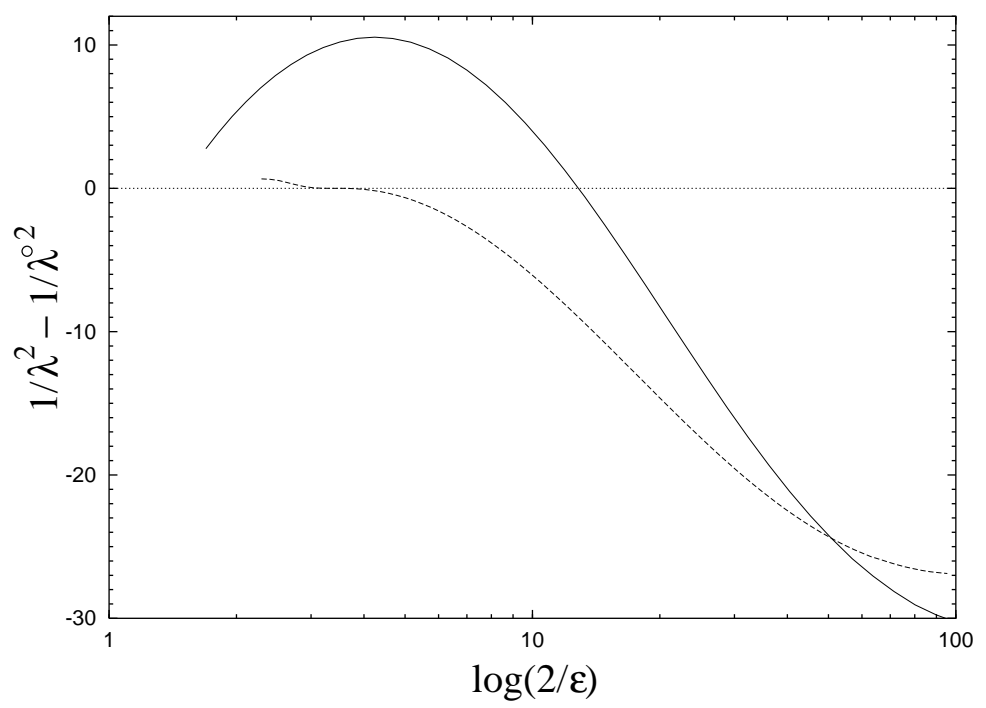

FIG. 4. The difference between the numerical solution and the analytic result, $\frac{1}{\tilde{\lambda}^{\circ}}=\frac{4}{\pi^{2}} \log ^{2} \frac{2}{\hat{\epsilon}}$ (solid line). For comparison, the $\hat{\delta}$-dependent behavior of the eigenvalue, given by (6.2), is indicated by the dashed line. Note the different scale used on the vertical axis, compared with Fig. 3 .

The numerical solution to (6.4) is presented in Fig. 3, and shows good agreement with the analytic result,

$$
\frac{1}{\widetilde{\lambda}^{\circ}}=\frac{4}{\pi^{2}} \log ^{2} \frac{2}{\hat{\epsilon}}=\frac{4}{\pi^{2}}\left(\log \frac{2}{\epsilon}+\log \frac{256 \mu^{6}}{\pi m_{D}^{5}}\right)^{2},
$$

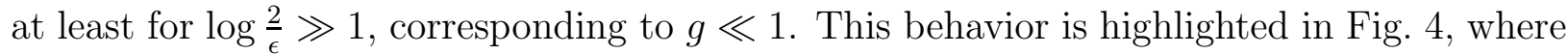
the difference between the numerical and analytical solutions is shown. Up to the linear log level, there is no discrepancy between the solutions; as they were obtained in different gauges, these results indeed support the gauge invariance of the critical temperature.

The behavior in Fig. A clearly indicates the presence of subleading terms, contributing below the linear log level. A large portion of this appears to be related to the treatment of the ultraviolet cutoff, taken as $\hat{\delta}=1$ in the analytical work. The numerical work, on the other hand, is suggestive of the presence of an effective cutoff near the Fermi surface, $\delta \approx \mu$, corresponding to $\hat{\delta}=(\pi / 256)\left(m_{D} / \mu\right)^{5}$. While any difference between these cases is formally seen from (6.3) to be down by three powers of the logarithm, it however contains a hidden enhancement due to the factor of $m_{D}^{5}$ entering into $\hat{\delta}$. Since $m_{D} \sim g \sim\left(\log \frac{2}{\epsilon}\right)^{-1}$ (using the zeroth order relation), the relevant expansion parameter is $\left(\log \hat{\delta} / \log \frac{2}{\hat{\epsilon}}\right) \sim(5 \log x) / x$ where $x=\log \frac{2}{\epsilon}$. Even for $x \approx 100$, which is the largest value we examined numerically, its cube is only down by about one percent.

For relevant values of $g$, which are of order one, the values of $x$ range from about 5 to 30 , in which case this sensitivity to the cutoff can lead to effects of order $10 \%$. On the other hand, for the nonlinear gap equation, this sensitivity appears much more pronounced; varying the ultraviolet cutoff between $\delta$ and $\mu \hat{\delta}$ can lead to as much as a factor of two or more change in the value of the zero temperature gap! 


\section{CONCLUSION.}

In this paper, we present an exact calculation of the transition temperature to the colorsuperconducting phase of $S U(N)_{c}$ QCD in the limit of high baryon density. To do this we cast the Fredholm integral equation that arises from the Dyson-Schwinger approach to di-quark scattering into a general eigenvalue problem. The leading order of the eigenvalue, found to be doubly logarithmic, gives rise to the non-BCS dependence of the exponent upon the coupling. We have developed a perturbation method which enables us to calculate the eigenvalue beyond the leading order in logarithm. The result, together with the contribution from the radiative correction of the quark propagator found in [31], leads to a complete determination of both the exponential and the pre-factor of the scaling formula for the transition temperature, (1.2), to the leading order of the running coupling constant. The transition temperature and the zero temperature gap are both suppressed relative to previous claims due to the effects of the quark self-energy. For $N=N_{f}=3$, these effects reduce the results by a significant factor of 0.17663 .

Using the one-loop formula for the running coupling constant at the chemical potential,

$$
g^{2}=\frac{12 \pi^{2}}{\left(\frac{11}{2} N-N_{f}\right) \log \frac{\mu}{\Lambda}}
$$

the behavior of the transition temperature is shown in Fig. 5 for a longitudinal pairing with zero angular momentum for $N=N_{f}=3$ and $\Lambda=200 \mathrm{MeV}$. We note that $g \simeq 1$ corresponds to $\mu \simeq 10^{6} \mathrm{MeV}$ and a transition temperature $k_{B} T_{C} \simeq 1.9 \mathrm{MeV}$. Our calculations become valid in the limit of high baryon density where the theory is weakly coupled, corresponding to the upward sloping arm on the right of Fig. 5 where $\mu>10^{6} \mathrm{MeV}$. However, there is some contention that such perturbative series are valid for $g \leq 4$, see [33] and the references therein. Extrapolating our results to $g \sim 3$ corresponds to $\mu \simeq 500 \mathrm{MeV}$ and $k_{B} T_{C} \simeq$ $3.7 \mathrm{MeV}$. This is the point at which the graph in Fig. 1 turns over and the behaviour becomes unphysical. This limit suggests that for $g>3$ strong coupling effects truly dominate and the transition temperature is determined by the mechanisms of chiral symmetry breaking and confinement but for $g<3$ our results may prove increasingly accurate.

The ratio between the transition temperature and the energy gap at zero temperature, $\Delta$, is an important number for superconductivity. This ratio has been calculated for zero angular momentum in [33] and the result is identical to that of BCS theory, i.e.

$$
\frac{\Delta}{k_{B} T_{C}}=\pi e^{-\gamma}
$$

Combining our formula for $k_{B} T_{C}^{J=0}$, (1.2), and this ratio we obtain that,

$$
\Delta=2 c_{1}^{\prime} \Delta_{0}=512 c_{1}^{\prime} \pi^{4}\left(\frac{2}{N_{f}}\right)^{\frac{5}{2}} \frac{\mu}{g^{5}} e^{-\sqrt{\frac{6 N}{N+1}} \frac{\pi^{2}}{g}},
$$

with $c_{1}^{\prime}$ given by (1.5) and $\Delta_{0}$ given by (1.3) and previously determined in [29. Apart from the new factor $c_{1}^{\prime}$, this is in agreement with the gap energy presented in 33] (we have highlighted the factor of 2 which was found numerically in 229 and by analytic means in 


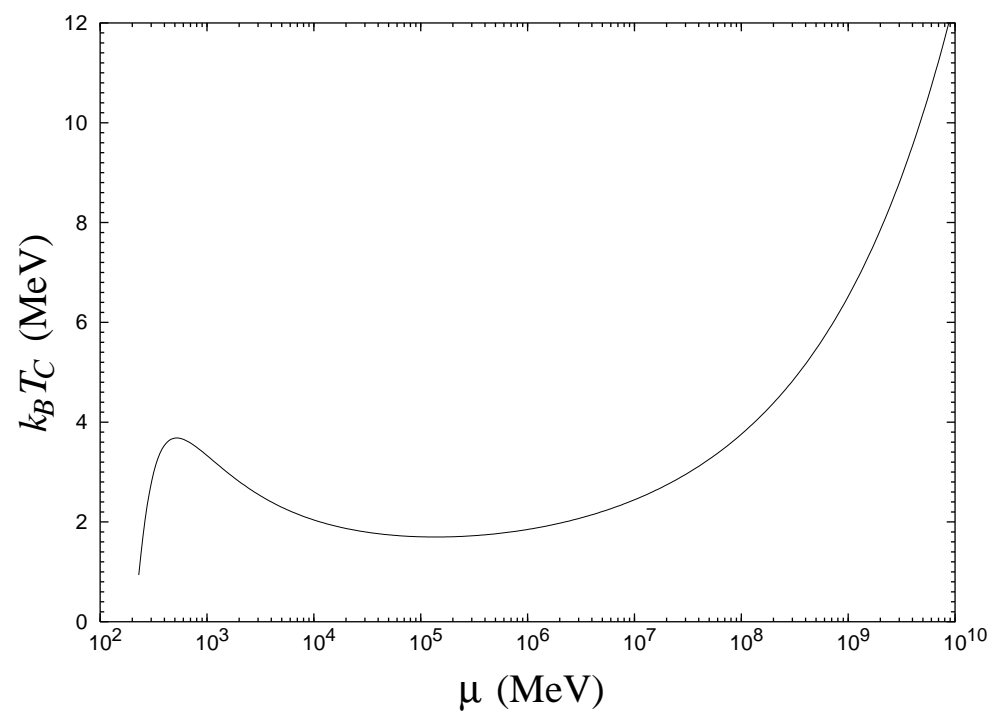

FIG. 5. The transition temperature, $k_{B} T_{C}$, as a function of the chemical potential, $\mu$, for $N=N_{f}=3$ and $\Lambda=200 \mathrm{MeV}$.

[33). What is significant, and perhaps somewhat surprising, is that the additional unknown pre-factor of $\mathcal{O}(1)$ present in all previous calculations of the zero temperature gap has been found to be exactly one to the leading order in the coupling.

We have extended the analysis to find the transition temperature for both longitudinal and transverse pairings for arbitrary angular momentum, $J \geq 1$. Relative to the longitudinal $J=0$ pairing these channels are suppressed by several orders of magnitude. As the temperature is reduced, once the highest transition temperature is reached the results for lower transition temperatures are invalid. However, if for some physical reason the $J=0$ longitudinal pair cannot form these results indicate that color-superconductivity may only occur at very low temperatures. Further investigation of the ratio (7.2) generalised to $J>0$ may produce interesting results for condensates of non-zero angular momentum.

\section{Acknowledgements.}

W.B. and H-C.R. would like to thank R. Pisarski and D. Rischke for stimulating discussions. The work of W.B. and H-C.R. is supported in part by the U.S. Department of Energy under grant DOE-91ER40651-TASKB. H-C. Ren's work is also supported in part by the Wiessman visiting professorship of Baruch College of CUNY.

\section{APPENDIX A: SINGLE GLUON EXCHANGE VERTEX.}

In this appendix, we set out some conventions and tabulate the single-gluon exchange vertex in all channels with $s= \pm$ and $s^{\prime}= \pm$. In Coulomb gauge, the relevant one gluon exchange contribution to (3.21) reads

$$
\Gamma_{s^{\prime}, s}^{A}\left(n^{\prime}, \vec{p}^{\prime} \mid n, \vec{p}\right)=-i \frac{g^{2}}{2}\left(1+\frac{1}{N}\right) D^{M}(\vec{k}, \omega)\left[\bar{u}\left(s_{1}^{\prime} \vec{p}^{\prime}\right) \gamma_{i} u\left(s_{1} \vec{p}\right)\right]\left[\bar{u}\left(-s_{2}^{\prime} \vec{p}^{\prime}\right) \gamma_{j} u\left(-s_{2} \vec{p}\right)\right]\left(\delta_{i j}-\hat{k}_{i} \hat{k}_{j}\right)
$$




$$
-i \frac{g^{2}}{2}\left(1+\frac{1}{N}\right) D^{E}(\vec{k}, \omega)\left[u^{\dagger}\left(s_{1}^{\prime} \vec{p}^{\prime}\right) u\left(s_{1} \vec{p}\right)\right]\left[u^{\dagger}\left(-s_{2}^{\prime} \vec{p}^{\prime}\right) u\left(-s_{2} \vec{p}\right)\right]
$$

where $\vec{k}=\vec{p}^{\prime}-\vec{p}$ and $\omega=\nu^{\prime}-\nu$. Using the Dirac equation $\left(-i \vec{\gamma} \cdot \vec{p}+\gamma_{4} p\right) u(\vec{p})=0$ and introducing the notation

$$
V_{s_{1}^{\prime}, s_{2}^{\prime} ; s_{1}, s_{2}}=\left[\bar{u}\left(s_{1}^{\prime} \vec{p}^{\prime}\right) \vec{\gamma} u\left(s_{1} \vec{p}\right)\right]\left[\bar{u}\left(-s_{2}^{\prime} \vec{p}^{\prime}\right) \vec{\gamma} u\left(-s_{2} \vec{p}\right)\right]
$$

and

$$
S_{s_{1}^{\prime}, s_{2}^{\prime} ; s_{1}, s_{2}}=\left[u^{\dagger}\left(s_{1}^{\prime} \vec{p}^{\prime}\right) u\left(s_{1} \vec{p}\right)\right]\left[u^{\dagger}\left(-s_{2}^{\prime} \vec{p}^{\prime}\right) u\left(-s_{2} \vec{p}\right)\right]
$$

we have

$$
\begin{aligned}
\Gamma_{s^{\prime}, s}^{A}\left(n^{\prime}, \vec{p}^{\prime} \mid n, \vec{p}\right)= & -i \frac{g^{2}}{2}\left(1+\frac{1}{N}\right) D^{M}(\vec{k}, \omega)\left[V_{s_{1}^{\prime}, s_{2}^{\prime} ; s_{1}, s_{2}}-\frac{\left(s_{1}^{\prime} p^{\prime}-s_{1} p\right)\left(s_{2}^{\prime} p^{\prime}-s_{2} p\right)}{\left(\vec{p}^{\prime}-\vec{p}\right)^{2}} S_{s_{1}^{\prime}, s_{2}^{\prime} ; s_{1}, s_{2}}\right] \\
& -i \frac{g^{2}}{2}\left(1+\frac{1}{N}\right) D^{E}(\vec{k}, \omega) S_{s_{1}^{\prime}, s_{2}^{\prime} ; s_{1}, s_{2}} .
\end{aligned}
$$

Our convention for gamma matrices is that $\left\{\gamma_{\mu}, \gamma_{\nu}\right\}=\delta_{\mu \nu}$ with each $\gamma_{\mu}$ hermitian and

$$
\gamma_{5}=\gamma_{1} \gamma_{2} \gamma_{3} \gamma_{4}=\left(\begin{array}{cc}
0 & -I \\
-I & 0
\end{array}\right)
$$

The coordinate system is chosen such that $\vec{p} \| \hat{z}$ and $\vec{p}^{\prime} \perp \hat{y}$ with $\vec{p}^{\prime} \cdot \hat{x}>0$. The azimuthal angle of $\vec{p}$ is assigned to be 0 and that of $-\vec{p}$ to be $\pi$. The polar angles of $\pm \vec{p}$ and $\pm \vec{p}^{\prime}$ are $\vec{p}:(0,0),-\vec{p}:(\pi, \pi), \vec{p}^{\prime}:(\theta, 0)$ and $-\vec{p}^{\prime}:(\pi-\theta, \pi)$. The chiral spinors at $\pm \vec{p}$ and $\pm \vec{p}^{\prime}$ are related via

$$
u(\vec{q})=e^{-\frac{i}{2} \sigma_{3} \phi} e^{-\frac{i}{2} \sigma_{2} \theta} e^{\frac{i}{2} \sigma_{3} \phi} u(\vec{p}),
$$

with $\vec{q}$ any one of $-\vec{p}$ and $\pm \vec{p}^{\prime}$ with $(\theta, \phi)$ the corresponding polar angles. For $\gamma_{5} u(\vec{p})=-u(\vec{p})$ we write

$$
u(\vec{p})=\frac{1}{\sqrt{2}}\left(\begin{array}{l}
1 \\
0 \\
1 \\
0
\end{array}\right) .
$$

Then the expressions for $V_{s_{1}^{\prime}, s_{2}^{\prime} ; s_{1}, s_{2}}$ and $S_{s_{1}^{\prime}, s_{2}^{\prime} ; s_{1}, s_{2}}$ follow and are listed in Table. I.

\section{APPENDIX B: FIRST ORDER CORRECTIONS FROM $\mathcal{K}_{1}^{(B)}$.}

In this appendix, we shall calculate the normalization constant of $|j\rangle$ due to discretecontinuous energy corrections, and calculate the contribution of $\mathcal{K}_{1}^{(b)}$ giving rise to (4.35). We begin with a simplification of the matrix elements of an operator of the structure

$$
\left\langle\nu^{\prime}, p^{\prime}|O| \nu, p\right\rangle=\frac{2 o\left(\nu^{\prime}, \nu\right)}{\sqrt{\nu^{2}+(p-\mu)^{2}} \sqrt{\nu^{\prime 2}+\left(p^{\prime}-\mu\right)^{2}}}
$$




\begin{tabular}{c|r|r}
\hline \hline$s_{1}^{\prime} s_{2}^{\prime}, s_{1} s_{2}$ & $V_{s_{1}^{\prime}, s_{2}^{\prime} ; s_{1}, s_{2}}$ & $S_{s_{1}^{\prime}, s_{2}^{\prime} ; s_{1}, s_{2}}$ \\
\hline,++++ & $\frac{3}{2}-\frac{1}{2} \cos \theta$ & $\frac{1}{2}+\frac{1}{2} \cos \theta$ \\
,+-++ & $\frac{1}{2} \sin \theta$ & $-\frac{1}{2} \sin \theta$ \\
,-+++ & $-\frac{1}{2} \sin \theta$ & $\frac{1}{2} \sin \theta$ \\
,--++ & $-\frac{3}{2}-\frac{1}{2} \cos \theta$ & $-\frac{1}{2}+\frac{1}{2} \cos \theta$ \\
,+++- & $-\frac{1}{2} \sin \theta$ & $\frac{1}{2} \sin \theta$ \\
,+-+- & $-\frac{1}{2}-\frac{1}{2} \cos \theta$ & $\frac{1}{2}+\frac{1}{2} \cos \theta$ \\
,-++- & $-\frac{1}{2}+\frac{1}{2} \cos \theta$ & $\frac{1}{2}-\frac{1}{2} \cos \theta$ \\
,--+- & $\frac{1}{2} \sin \theta$ & $\frac{1}{2} \sin \theta$ \\
,++-+ & $\frac{1}{2} \sin \theta$ & $-\frac{1}{2} \sin \theta$ \\
,+--+ & $-\frac{1}{2}+\frac{1}{2} \cos \theta$ & $\frac{1}{2}-\frac{1}{2} \cos \theta$ \\
,-+-+ & $-\frac{1}{2}+\frac{1}{2} \cos \theta$ & $\frac{1}{2}+\frac{1}{2} \cos \theta$ \\
,---+ & $-\frac{1}{2} \sin \theta$ & $-\frac{1}{2} \sin \theta$ \\
,++-- & $-\frac{3}{2}-\frac{1}{2} \cos \theta$ & $-\frac{1}{2}+\frac{1}{2} \cos \theta$ \\
,+--- & $\frac{1}{2} \sin \theta$ & $-\frac{1}{2} \sin \theta$ \\
,-+-- & $-\frac{1}{2} \sin \theta$ & $\frac{1}{2} \sin \theta$ \\
,---- & $\frac{3}{2}-\frac{1}{2} \cos \theta$ & $\frac{1}{2}+\frac{1}{2} \cos \theta$ \\
\hline \hline
\end{tabular}

TABLE I. The factors $V$ and $S$ for all combinations of incoming and outgoing quarks above and below the Fermi Sea.

For a function $\langle\nu, p \mid \psi\rangle$ pertaining to the ket vector $|\psi\rangle$, we introduce the function

$$
\langle\nu \mid \psi\rangle=\sqrt{2} \int_{0}^{\infty} d p \frac{\langle\nu, p \mid \psi\rangle}{\sqrt{\nu^{2}+(p-\mu)^{2}}}
$$

so that

$$
\left\langle\psi^{\prime}|O| \psi\right\rangle=\sum_{n=0}^{\infty} \frac{1}{\left(n^{\prime}+\frac{1}{2}\right)\left(n+\frac{1}{2}\right)}\left\langle\psi^{\prime} \mid \nu_{n^{\prime}}\right\rangle O\left(\nu_{n^{\prime}}, \nu_{n}\right)\left\langle\nu_{n} \mid \psi\right\rangle
$$

Note that we have extended the lower limit of the $p$ and $p^{\prime}$ integrals to $-\infty$; the correction introduced is beyond the order of interest. The same simplification extends to the next appendix as well.

Given $\mathcal{K}_{1}^{(b)}$ in Eqn. (4.33), we wish to evaluate the contribution

$$
\left\langle\tilde{0}\left|\mathcal{K}_{1}^{(b)}\right| \tilde{0}\right\rangle=\frac{\left\langle 0_{c}\left|\log \frac{1}{\hat{\nu}_{>}}\right| 0_{c}\right\rangle}{\left\langle 0_{c} \mid 0_{c}\right\rangle}-\frac{1}{\widetilde{\lambda}_{0}^{\circ 2}} .
$$

Note that while the eigenfunction,

$$
\left\langle\nu \mid 0_{c}\right\rangle=\chi_{0}(\nu)=2 \sqrt{\frac{t}{\pi}} \sin \left(t \log \frac{1}{\hat{\nu}}\right),
$$

where 


$$
t=\frac{\pi}{2 \log \frac{2}{\hat{\epsilon}}} \ll 1
$$

is properly normalized in the continuum norm, this may no long be the case under the

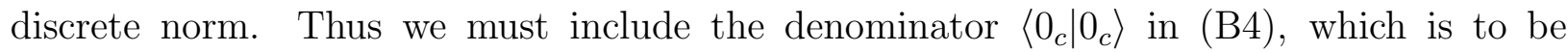
calculated with discrete energy variables.

Using (B5), this discrete norm of the zeroth order eigenfunction reads,

$$
\left\langle 0_{c} \mid 0_{c}\right\rangle=\frac{4 t}{\pi} \sum_{n=0}^{N_{0}} \frac{\hat{\epsilon}}{\hat{\nu}_{n}} \sin ^{2}\left(t \log \frac{1}{\hat{\nu}_{n}}\right),
$$

where

$$
\hat{\nu}_{n}=\left(n+\frac{1}{2}\right) \hat{\epsilon}
$$

and $N_{0} \gg 1$ is the discrete cutoff corresponding to the ultraviolet cutoff, $\hat{\delta}$, in the continuum: $\hat{\delta}=\left(N_{0}+\frac{1}{2}\right) \hat{\epsilon}$.

Evaluation of this sum may be performed in terms of the generalized zeta-function, [37],

$$
\zeta(s, a)=\sum_{n=0}^{\infty} \frac{1}{(n+a)^{s}},
$$

resulting in,

$$
\begin{aligned}
\left\langle 0_{c} \mid 0_{c}\right\rangle=\frac{2 t}{\pi}\left\{\zeta\left(1+0^{+}, \frac{1}{2}\right)-\zeta\left(1+0^{+}, N_{0}+\frac{3}{2}\right)\right. & \\
& \left.-\operatorname{Re} \hat{\epsilon}^{-2 i t}\left[\zeta\left(1+2 i t, \frac{1}{2}\right)-\zeta\left(1+2 i t, N_{0}+\frac{3}{2}\right)\right]\right\} .
\end{aligned}
$$

Using the asymptotic formula for $t \rightarrow 0$,

$$
\zeta(1+2 i t, a)=-i \frac{\Gamma(a)}{2 t \Gamma(a+i t)}+\mathcal{O}(t),
$$

which follows the integral representation of $\zeta(s, a)$, we obtain that

$$
\zeta\left(1+2 i t, \frac{1}{2}\right) \simeq-i \frac{1}{2 t}-\psi\left(\frac{1}{2}\right)
$$

with $\psi(z)$ the logarithmic derivative of $\Gamma(z)$ and

$$
\zeta\left(1+2 i t, N_{0}+\frac{3}{2}\right) \simeq-\frac{i}{2 t}\left(N_{0}+\frac{3}{2}\right)^{-2 i t}
$$

Therefore, we end up with

$$
\left\langle 0_{c} \mid 0_{c}\right\rangle \simeq 1-\frac{4 t}{\pi}\left(\psi\left(\frac{1}{2}\right)+\log 2\right)=1+2(\gamma+\log 2) \log ^{-1} \frac{2}{\hat{\epsilon}}
$$

where we have used $\psi\left(\frac{1}{2}\right)=-\gamma-2 \log 2$.

Evaluating the matrix element of the kernel is more difficult. We start with the expression 


$$
\left\langle 0_{c}\left|\log \frac{1}{\hat{\nu}_{>}}\right| 0_{c}\right\rangle=\frac{4 t}{\pi} \sum_{n=0}^{N_{0}} \frac{\hat{\epsilon}}{\hat{\nu}_{n}} \sum_{m=0}^{N_{0}} \frac{\hat{\epsilon}}{\hat{\nu}_{m}} \sin \left(t \log \frac{1}{\hat{\nu}_{n}}\right)\left[\log \frac{1}{\hat{\nu}_{>}}\right] \sin \left(t \log \frac{1}{\hat{\nu}_{m}}\right) .
$$

In order to resolve $\hat{\nu}_{>}$we split the double sum, making use of the symmetry under $n \leftrightarrow m$ interchange, and obtain

$$
\left\langle 0_{c}\left|\log \frac{1}{\hat{\nu}_{>}}\right| 0_{c}\right\rangle=-\frac{8 t}{\pi} \sum_{n=0}^{N_{0}} \frac{\hat{\epsilon}}{\hat{\nu}_{n}} S_{n}(t) \frac{d}{d t} \cos \left(t \log \frac{1}{\hat{\nu}_{n}}\right),
$$

where

$$
S_{n}(t)=\sum_{m=0}^{n} \frac{\hat{\epsilon}}{\hat{\nu}_{m}} \sin \left(t \log \frac{1}{\hat{\nu}_{m}}\right)=\operatorname{Im}\left\{\hat{\epsilon}^{-i t}\left[\zeta\left(1+i t, \frac{1}{2}\right)-\zeta\left(1+i t, n+\frac{3}{2}\right)\right]\right\} .
$$

Inserting (B17) into (B16), we find

$$
\begin{aligned}
\left\langle 0_{c}\left|\log \frac{1}{\hat{\nu}_{>}}\right| 0_{c}\right\rangle= & \frac{2 i t}{\pi}\left\{\left[\hat{\epsilon}^{-i t} \zeta\left(1+i t, \frac{1}{2}\right)-\hat{\epsilon}^{i t} \zeta\left(1-i t, \frac{1}{2}\right)\right]\right. \\
& \times \frac{\partial}{\partial t}\left[\hat{\epsilon}^{-i t}\left(\zeta\left(1+i t, \frac{1}{2}\right)-\zeta\left(1+i t, N_{0}+\frac{3}{2}\right)\right)+\hat{\epsilon}^{i t}\left(\zeta\left(1-i t, \frac{1}{2}\right)-\zeta\left(1-i t, N_{0}+\frac{3}{2}\right)\right)\right] \\
- & \hat{\epsilon}^{-i t} \frac{\partial}{\partial t}\left[\hat{\epsilon}^{-i t}\left(Z\left(t^{\prime}, t ; \frac{1}{2}\right)-Z\left(t^{\prime}, t ; N_{0}+\frac{3}{2}\right)\right)+\hat{\epsilon}^{i t}\left(Z\left(t^{\prime},-t ; \frac{1}{2}\right)-Z\left(t^{\prime},-t ; N_{0}+\frac{3}{2}\right)\right)\right]_{t^{\prime}=t} \\
+ & \left.+\hat{\epsilon}^{i t} \frac{\partial}{\partial t}\left[\hat{\epsilon}^{-i t}\left(Z\left(-t^{\prime}, t ; \frac{1}{2}\right)-Z\left(-t^{\prime}, t ; N_{0}+\frac{3}{2}\right)\right)+\hat{\epsilon}^{i t}\left(Z\left(-t^{\prime},-t ; \frac{1}{2}\right)-Z\left(-t^{\prime},-t ; N_{0}+\frac{3}{2}\right)\right)\right]_{t^{\prime}=t}\right\},
\end{aligned}
$$

where,

$$
\begin{aligned}
Z\left(t^{\prime}, t ; a\right) & =\sum_{n=0}^{\infty} \frac{1}{(n+a)^{1+i t}} \zeta\left(1+i t^{\prime}, 1+a+n\right) \\
& =\frac{1}{\Gamma\left(1+i t^{\prime}\right) \Gamma(1+i t)} \int_{0}^{\infty} d x \int_{0}^{\infty} d y \frac{x^{i t^{\prime}} y^{i t} e^{-x} e^{-a(x+y)}}{\left(1-e^{-x}\right)\left(1-e^{-x-y}\right)} .
\end{aligned}
$$

Changing the integration variables to $u=1-e^{-x}$ and $v=1-e^{-x-y}$, we find for $t \rightarrow 0$ and $t^{\prime} \rightarrow 0$ that

$$
Z\left(t^{\prime}, t ; a\right)=-\frac{1}{t^{\prime}\left(t+t^{\prime}\right)} \frac{\Gamma(a)}{\Gamma\left(a+i\left(t+t^{\prime}\right)\right)}+\mathcal{O}(1)
$$

Therefore

$$
Z\left(t^{\prime}, t ; \frac{1}{2}\right) \simeq-\frac{1}{t^{\prime}\left(t+t^{\prime}\right)}+\frac{i}{t^{\prime}} \psi\left(\frac{1}{2}\right)
$$

and

$$
Z\left(t^{\prime}, t ; N_{0}+\frac{3}{2}\right) \simeq-\frac{1}{t^{\prime}\left(t+t^{\prime}\right)}\left(N_{0}+\frac{3}{2}\right)^{-i\left(t^{\prime}+t\right)}
$$


Upon substituting the above expressions into (B18), and expanding for $t \ll 1$, after some manipulations we obtain

$$
\left\langle 0_{c}\left|\log \frac{1}{\hat{\nu}_{>}}\right| 0_{c}\right\rangle \simeq \frac{1}{t^{2}}\left[1-\frac{8 t}{\pi}\left(\psi\left(\frac{1}{2}\right)+\log 2\right)\right]=\frac{4}{\pi^{2}} \log ^{2} \frac{2}{\hat{\epsilon}}\left[1+4(\gamma+\log 2) \log ^{-1} \frac{2}{\hat{\epsilon}}\right] .
$$

Finally, after combining this with the discrete normalization, (B14), and subtracting out the zeroth order eigenvalue, we find the claimed result,

$$
\left\langle\tilde{0}\left|\mathcal{K}_{1}^{(b)}\right| \tilde{0}\right\rangle=\frac{4}{\pi^{2}}\left[2(\gamma+\log 2) \log \frac{2}{\hat{\epsilon}}+\mathcal{O}(1)\right] .
$$

In addition, we have also calculated the general inner products $\left\langle j_{c} \mid j_{c}^{\prime}\right\rangle$ and the matrix element $\left\langle 0_{c}\left|\log \frac{1}{\hat{\nu}_{>}}\right| j_{c}\right\rangle$, and found that

$$
\begin{aligned}
& \left\langle j_{c} \mid j_{c}\right\rangle=\left\langle 0_{c} \mid 0_{c}\right\rangle \\
& \left\langle j_{c} \mid j_{c}^{\prime}\right\rangle=\mathcal{O}\left(\log ^{-1} \frac{2}{\hat{\epsilon}}\right) \quad \text { for } j \neq j^{\prime}
\end{aligned}
$$

and

$$
\left\langle 0_{c}\left|\log \frac{1}{\hat{\nu}_{>}}\right| j_{c}\right\rangle=\mathcal{O}\left(\log \frac{2}{\hat{\epsilon}}\right)
$$

for $j \neq 0$.

\section{APPENDIX C: THE DISCRETE KERNEL IN THE CONTINUUM LIMIT}

Here we demonstrate that in the continuum limit $\mathcal{K}_{0}$ indeed leads to the continuum approximate form (4.11). To leading order, there is no distinction between $\left.|j\rangle, j_{c}\right\rangle$ and $|\tilde{j}\rangle$,

nor between the eigenvalues of the continuous or discrete theories. Thus we are interested in the quantity,

$$
\left\langle\nu_{n}\left|\mathcal{K}_{0}\right| \nu_{n^{\prime}}\right\rangle=\sum_{j} \tilde{\lambda}_{j}^{\circ-2}\left\langle\nu_{n} \mid \tilde{j}\right\rangle\left\langle\tilde{j} \mid \nu_{n^{\prime}}\right\rangle \simeq \sum_{j} \widetilde{\lambda}_{j}^{\circ-2}\langle\nu \mid j\rangle\left\langle j \mid \nu^{\prime}\right\rangle
$$

where the last expression on the right hand side contains continuous energy variables and the last inequality holds at leading order only. Since $\langle\nu \mid j\rangle=\chi(\nu)$, using equation (4.13), we may re-write (C1) as,

$$
\sum_{j} \widetilde{\lambda}_{j}^{\circ-2}\langle\nu \mid j\rangle\left\langle j \mid \nu^{\prime}\right\rangle=\frac{1}{\pi^{2}} \log \frac{2}{\hat{\epsilon}}\left[I\left(\theta_{\nu}-\theta_{\nu^{\prime}}\right)-I\left(\theta_{\nu}+\theta_{\nu^{\prime}}\right)\right],
$$

where

$$
I(\alpha) \equiv \sum_{j=0}^{\infty} \frac{1}{\left(j+\frac{1}{2}\right)^{2}} \cos \left[\left(j+\frac{1}{2}\right) \alpha\right], \quad-\pi \leq \alpha \leq \pi,
$$

and 


$$
\theta_{\nu}=\pi \frac{\log 1 / \hat{\nu}}{\log 2 / \hat{\epsilon}}, \quad 0<\theta_{\nu} \leq \pi
$$

To evaluate $I(\alpha)$ we consider its derivative,

$$
\frac{d I}{d \alpha}=-\operatorname{Im} \sum_{j=0}^{\infty} \frac{1}{j+\frac{1}{2}} e^{i\left(j+\frac{1}{2}\right) \alpha}=-\operatorname{Im} \log \left[\frac{1+e^{i \alpha / 2}}{1-e^{i \alpha / 2}}\right]=2 m \pi-\frac{\pi}{2},
$$

where $m$ is an integer, yet to be determined. The condition $\left.\frac{d I}{d \alpha}\right|_{\alpha=\pi}=-\frac{\pi}{2}$ then fixes $m$ to be zero and $I(\alpha=\pi)=0$ fixes the constant of integration to give $I(\alpha)$ from (C5). Since $I(\alpha)$ is an even and antiperiodic function, we find,

$$
I(\alpha)=\frac{\pi}{2}(\pi-|\alpha|), \quad-2 \pi \leq \alpha \leq 2 \pi .
$$

Substituting (C6) into (C2) then yields,

$$
\left\langle\nu\left|\mathcal{K}_{0}\right| \nu^{\prime}\right\rangle= \begin{cases}\log 1 / \hat{\nu}^{\prime} & \text { if } \hat{\nu}^{\prime}>\hat{\nu} \\ \log 1 / \hat{\nu} & \text { if } \hat{\nu}^{\prime}<\hat{\nu}\end{cases}
$$

which is in direct agreement with the leading order kernel written in (4.11).

\section{APPENDIX D: FIRST ORDER CORRECTIONS FROM $\mathcal{K}_{1}^{(A)}$.}

In this appendix, we calculate the first order perturbation from $\mathcal{K}_{1}^{(a)}$. On splitting the discrete energy sums resulting from evaluation of the matrix element into diagonal and off-diagonal parts,

$$
\left\langle 0\left|\mathcal{K}_{1}^{(a)}\right| 0\right\rangle=I+J
$$

we have

$$
I=\frac{1}{\beta^{2}} \sum_{n=0}^{N_{0}} \int_{0}^{\infty} d p \int_{0}^{\infty} d p^{\prime} \mathcal{K}_{1}^{(a)}\left(n, p \mid n, p^{\prime}\right)\langle\tilde{0} \mid n, p\rangle\left\langle n, p^{\prime} \mid \tilde{0}\right\rangle
$$

and

$$
J=\frac{1}{\beta^{2}} \sum_{n=0}^{N_{0}} \sum_{n^{\prime}=0}^{N_{0}}\left(1-\delta_{n n^{\prime}}\right) \int_{0}^{\infty} d p \int_{0}^{\infty} d p^{\prime} \mathcal{K}_{1}^{(a)}\left(n, p \mid n^{\prime}, p^{\prime}\right)\langle\tilde{0} \mid n, p\rangle\left\langle n^{\prime}, p^{\prime} \mid \tilde{0}\right\rangle,
$$

where $N_{0} \gg 1$, the discrete cutoff, is given by $\delta=\left(N_{0}+\frac{1}{2}\right) \epsilon$ with $\delta$ the ultraviolet cutoff introduced in section IV.

For the first term, using (4.8), we find that

$$
I=\frac{8 t}{\pi \beta^{2}} \sum_{n=0}^{N_{0}} \int_{0}^{\infty} d p \int_{0}^{\infty} d p^{\prime} \frac{3 \log \frac{p+p^{\prime}}{\left|p-p^{\prime}\right|}-2 \log \frac{1}{\hat{\nu}_{n}}}{\sqrt{\left[\nu_{n}^{2}+(p-\mu)^{2}\right]\left[\nu_{n}^{2}+\left(p^{\prime}-\mu\right)^{2}\right]}} \sin ^{2}\left(t \log \frac{1}{\hat{\nu}_{n}}\right)
$$


with $t=\frac{\pi}{2 \log \frac{2}{\hat{\epsilon}}}$. For $\epsilon \ll \mu$, (D4) can be approximated by

$$
\begin{aligned}
I & \simeq \frac{2 t}{\pi^{3}} \sum_{n=0}^{\infty} \frac{1}{\left(n+\frac{1}{2}\right)^{2}} \sin ^{2}\left(t \log \frac{1}{\hat{\nu}_{n}}\right) \int_{-\infty}^{\infty} d x \int_{-\infty}^{\infty} d y \frac{1}{\left(x^{2}+1\right)\left(y^{2}+1\right)} \\
& \times\left[3 \log \frac{2 \mu}{|x-y|\left(n+\frac{1}{2}\right) \epsilon}-2 \log \frac{1}{\left(n+\frac{1}{2}\right) \hat{\epsilon}}\right] \\
& \simeq \frac{2 t}{\pi} \log \frac{2 \mu}{\epsilon} \sum_{n=0}^{\infty} \frac{1}{\left(n+\frac{1}{2}\right)^{2}} \sin ^{2}\left(t \log \frac{1}{\hat{\nu}_{n}}\right) \\
& =\mathcal{O}(1)
\end{aligned}
$$

for $t \rightarrow 0$, since $\log \frac{2 \mu}{\epsilon} \sim t^{-1}$ and the infinite series converges.

Next, instead of calculating $J$ directly, we evaluate the more general expression,

$$
J_{m}=\frac{1}{\beta^{2}} \sum_{n=0}^{N_{0}} \sum_{n^{\prime}=0}^{N_{0}}\left(1-\delta_{n n^{\prime}}\right) \int_{0}^{\infty} d p \int_{0}^{\infty} d p^{\prime} \mathcal{K}_{1}^{(a)}\left(n, p \mid n^{\prime}, p^{\prime}\right)\langle\tilde{m} \mid n, p\rangle\left\langle n^{\prime}, p^{\prime} \mid \tilde{m}\right\rangle,
$$

so that $J=J_{m=0}$. It follows from (4.32) for $\epsilon \ll \mu$, that

$$
\begin{aligned}
J_{m}= & \frac{2 t}{\pi} \sum_{n=0}^{N_{0}} \sum_{n^{\prime}=0}^{N_{0}}\left(1-\delta_{n n^{\prime}}\right) \frac{1}{\left(n+\frac{1}{2}\right)\left(n^{\prime}+\frac{1}{2}\right)}\left(\log \frac{1}{\left|\hat{\nu}_{n}-\hat{\nu}_{n^{\prime}}\right|}+\log \frac{1}{\left|\hat{\nu}_{n}+\hat{\nu}_{n^{\prime}}\right|}-2 \log \frac{1}{\hat{\nu}_{>}}\right) \\
& \quad \times \sin \left[(2 m+1) t \log \frac{1}{\hat{\nu}_{n}}\right] \sin \left[(2 m+1) t \log \frac{1}{\hat{\nu}_{n^{\prime}}}\right] \\
= & \frac{4 t}{\pi} \sum_{n=1}^{N_{0}} \sum_{n^{\prime} \neq 0}^{n-1} \frac{1}{\left(n+\frac{1}{2}\right)\left(n^{\prime}+\frac{1}{2}\right)} \sum_{l=1}^{\infty} \frac{1}{l}\left(\frac{\hat{\nu}_{n^{\prime}}}{\hat{\nu}_{n}}\right)^{2 l} \sin \left[(2 m+1) t \log \frac{1}{\hat{\nu}_{n}}\right] \sin \left[(2 m+1) t \log \frac{1}{\hat{\nu}_{n^{\prime}}}\right] \\
\simeq & \frac{4 t}{\pi} \sum_{l=1}^{\infty} \frac{1}{l} \int_{\frac{\hat{\epsilon}}{2}}^{\delta} \frac{d \hat{\nu}}{\hat{\nu}} \int_{\frac{\hat{\epsilon}}{2}}^{\delta} \frac{d \hat{\nu}^{\prime}}{\hat{\nu}^{\prime}}\left(\frac{\hat{\nu}^{\prime}}{\hat{\nu}}\right)^{2 l} \sin \left[(2 m+1) t \log \frac{1}{\hat{\nu}}\right] \sin \left[(2 m+1) t \log \frac{1}{\hat{\nu}^{\prime}}\right] \\
= & \frac{4 t}{\pi} \sum_{l=1}^{\infty} \frac{1}{l} \int_{\log \frac{1}{\delta}}^{\log \frac{2}{\hat{\epsilon}}} d x \int_{\log \frac{1}{\delta}}^{x} d x^{\prime} e^{-2 l\left(x-x^{\prime}\right)} \sin [(2 m+1) t x] \sin \left[(2 m+1) t x^{\prime}\right] \\
& \sim \frac{4 t}{\pi} \sum_{l=1}^{\infty} \frac{1}{l} \int_{0}^{\log \frac{2}{\hat{\epsilon}}} d x \int_{0}^{x} d x^{\prime} e^{-2 l\left(x-x^{\prime}\right)} \sin [(2 m+1) t x] \sin \left[(2 m+1) t x^{\prime}\right] \\
\simeq & \frac{1}{2} \sum_{l=1}^{\infty} \frac{1}{l^{2}+\left(m+\frac{1}{2}\right)^{2} t^{2}}=\frac{\pi^{2}}{12}
\end{aligned}
$$

as $t \rightarrow 0$. Therefore $J=\mathcal{O}(1)$ and no linearly logarithmic terms result from $\left\langle 0\left|\mathcal{K}_{1}^{(a)}\right| 0\right\rangle=$ $\mathcal{O}(1)$. On the other hand, as $m \rightarrow \infty$ at a fixed $t, J_{m} \sim 1 / m$. Since $J_{m}$ is proportional to the first order shift of the $\frac{1}{\lambda_{m}^{2}}$ given by (4.14), the large $m$ behavior of $J_{m}$ explains the weak logarithmic singularity of the Fredholm kernel. 


\section{REFERENCES}

[1] B. Barrois, Nucl. Phys. B129 (1977) 390.

[2] S. Frautschi, Proceedings of the Workshop on Hadronic Matter at Extreme Energy Density, N. Cabibbo, Editor, Erice, Italy, 1978.

[3] D. Bailin and A. Love, Phys. Rep. 107 (1984) 325, and references therein.

[4] M. Alford, K. Rajagopal and F. Wilczek, Phys. Lett. B422 (1998) 247-256.

[5] R. Rapp, T. Schafer, E.V. Shuryak and M. Velkovsky, Phys. Rev. Lett. 81 (1998) 53-56.

[6] M. Alford, K. Rajagopal and F. Wilczek, Nucl. Phys B537 (1999) 443-458.

[7] T. Schäfer and F. Wilczek, Phys. Rev. Lett. 82 (1999) 3956-3959.

[8] M. Alford, J. Berges and K. Rajagopal, hep-ph/9903502.

[9] T. Shafer and F. Wilczek, Phys. Rev. D60 (1999) 074014.

[10] N. Evans, J. Hormuzdiar, S.D.H. Hsu and M. Schwetz, hep-ph/9910313.

[11] M. Alford, J. Berges and K. Rajagopal, hep-ph/9908235.

[12] D.K. Hong, M. Rho and I. Zahed, Phys. Lett. B468 (1999) 261.

[13] R. Casalbuoni and R. Gatto, Phys. Lett. B464 (1999) 111; hep-ph/9909419; hep-ph/9911223.

[14] M. Rho, A. Wirzba, I. Zahed, hep-ph/9910550.

[15] J. Berges and K. Rajagopal, Nucl. Phys. B538 (1999) 215.

[16] G. Carter and D. Diakonov, Phys. Rev. D60 (1999) 016004.

[17] R.D. Pisarski, D.H. Rischke, Phys. Rev. Lett 83 (1999) 37-40.

[18] K. Rajagopal, hep-ph/9908360.

[19] P.F. Bedaque, hep-ph/9910247.

[20] M. Alford, J. Berges and K. Rajagopal, hep-ph/9910254.

[21] D. Blaschke, D.M. Sedrakian and K.M. Shahabasyan, astro-ph/9904395; astro-ph/9911349.

[22] D. Blaschke, T. Klaehn and D.N. Voskresensky, astro-ph/9908334.

[23] M. Matsuzaki, hep-ph/9910541.

[24] R. Rapp, T. Schafer, E.V. Shuryak and M. Velkovsky, submitted to Ann. Phys., hep-ph/9904353.

[25] N. Evans, S.D.H. Hsu and M. Schwetz, Nucl. Phys. B551 (1999) 275.

[26] N. Evans, S.D.H. Hsu and M. Schwetz, Phys. Lett. B449 (1999) 281.

[27] T. Shafer and F. Wilczek, Phys. Lett. B450 (1999) 325.

[28] D. T. Son, Phys. Rev. D59 (1999) 094019.

[29] T. Shafer and F. Wilczek, Phys. Rev. D60 (1999) 114033.

[30] R.D. Pisarski, D.H. Rischke, nucl-th/9907041.

[31] W.E. Brown, J.T. Liu and H-C.Ren, hep-ph/9908248, to appear in Phys. Rev. D.

[32] D.H. Hsu and M. Schwetz, hep-ph/9908310.

[33] R.D. Pisarski, D.H. Rischke, nucl-th/9910056.

[34] L.P. Gor'kov and T.K. Melik-Barkhudarov, Soviet Physics JETP 13 (1961) 1018.

[35] M. Le Bellac, Thermal Field Theory, Cambridge University Press, 1996.

[36] M.L. Goldberg, M.T. Grisaru, S.W. MacDowell and D.Y. Wong, Phys. Rev. 120 (1960) 2250.

[37] E.T. Whittaker and G.N. Watson, A Course of Modern Analysis, Cambridge University Press, $4^{\text {th }}$ edition, 1962. 